\title{
GAS EFFECT FOR OBLIQUE AND CONICAL SHOCK WAVES AT HIGH TEMPERATURE
}

\author{
Toufik Yahiaoui ${ }^{1}$, Toufik Zebbiche ${ }^{2, *}$, AbDerrazak Allali ${ }^{1}$ \\ AND MOHAMED BOUn-JAD ${ }^{1}$
}

\begin{abstract}
The work focuses to develop a new numerical calculation program for determining the gas effect at high temperature instead air on the calculation of the oblique and conical shock waves parameters and make applications for various external and internal aerodynamics problems like, the calculation of the suitable intake adaptation parameters, dihedron and cone wave drag, aerodynamic coefficients of a pointed supersonic airfoil and oblique shock reflection without forgetting others no less important like the detonation propulsion and the dust explosion applications, where the high temperature gas effect is very important. All this for future aerodynamics (gas dynamics) like the phenomenon of climate change in the near and far future because of the enlargement progressive of the layer ozone hole which will lead to an increase in the temperature of the ambient medium, and by the environment pollution by the shining of the waste which will cause a new decomposition of gases from the ambient environment. Another interesting application for actual aerodynamics (gas dynamics) is the performance of tests in wind tunnels supplied by a combustion chamber making a reaction of gases giving a gas with new thermodynamics parameters which is not necessarily air. To make a calculation, the selected gases are $\mathrm{H}_{2}, \mathrm{O}_{2}, \mathrm{~N}_{2}, \mathrm{CO}, \mathrm{CO}_{2}, \mathrm{H}_{2} \mathrm{O}, \mathrm{NH}_{3}, \mathrm{CH}_{4}$ and air. All shock parameters depend on the stagnation temperature, upstream Mach number, the thermodynamics of the used gas, dihedron and cone deviation and others parameters. The specific heat at constant pressure varies with the temperature and the selected gas. Gas is still considered as perfect. It is calorically imperfect, and thermally perfect, less than the molecules dissociation threshold. A comparison between the parameters of each gas and air is presented to choose the suitable gas witch giving good performances as required by design parameters instead air.
\end{abstract}

Mathematics Subject Classification. 35Q92.

Received March 31, 2020. Accepted September 18, 2020.

\section{INTRODUCTION}

Gases play an important role in the aerodynamics (gas dynamics) for aeronautical and aerospace's vehicles. They influence the behavior of the supersonic flows and in particular on all thermodynamics and physical

Keywords and phrases: Supersonic flow, subsonic flow, high temperature, thermodynamics ratios, normal shock wave, oblique shock wave, gas, conical shock wave, intake adaptation, shock reflection, aerodynamic coefficients, pointed airfoil, specific heat at constant pressure, detonation propulsion, dust explosion, error.

${ }^{1}$ Industrial Technology Studies and Research Laboratory (Ex Aircraft Laboratory), Department of Mechanical Engineering, Faculty of Technology, University of Blida 1, Algeria.

2 Institute of Aeronautics and Space Studies, University of Blida 1, BP 270 Blida 09000, Algeria.

* Corresponding author: z_toufik270169@yahoo.fr 
parameters. The $N S W, O S W$ and $C S W$ are a physical phenomenon appeared in nature to only supersonic flows when conditions are applied $[2,3,13,25]$.

In a supersonic nozzle when $P_{E}<P_{a}$, a $N S W$ will develop in interior of the nozzle. The position and the parameters through the shock depend on $P_{E} / P_{a}$. In this case, the flow is subsonic, and it will affect the $C_{F}$ of the nozzle $[22,25]$.

The attached $O S W$ and $C S W$ will respectively appear around the two-dimensional and axisymmetric pointed obstacles in a supersonic flow $[18,19,27]$. They have a very small thickness, so that they can be considered as mathematical discontinuity $[2,3]$. Therefore, all physical parameters have a discontinuity through the shock except the total temperature.

The work done by $[1-3,13,18,19,22,24,25,27]$ for the $N S W, O S W$ and $C S W$ parameters are made by a calorically perfect ideal gas, i.e. the $C_{P}$ is constant, and does not depend on the temperature. It gives good results for low $T_{0}, M_{1}$ and $\varphi$, which does not, exceed $240 \mathrm{~K}, 2.00$ and $10^{\circ}$ respectively. This model for gas is called by $P G$ model.

Air is frequently used as a gas in external and internal propulsion flows for all aeronautical, aerospace or even wind tunnel applications, especially for shock waves calculation, as it exists in quantity in nature $[1-3,12,13,16-19,22,24,25,27,31]$.

In $[5,7,8]$, the authors have developed the first study on the application of the propulsion gas effect on the conception of supersonic nozzle $M L N$ 2D [5], $M L N$ axisymmetric [7] and $P N$ 2D [8] to HT. For the studies in $[5,7,8]$ to be carried out, reference [9] always deals with the gas effect on the Prandtl-Meyer function at $H T$.

A study on the propellant gas effect at $H T$ on the parameters through a $N S W$ in non-adaptation regime of a supersonic nozzle is the goal of [9].

Others important applications for oblique shock wave are extended to present some problems for oblique detonation process. Modeling aluminum particle-air detonation is very difficult because the combustion phenomenon is shock-induced, and there are multi-phase heat release and transfer in supersonic flows. The reference [28] presents 2D cellular Aluminum particle-air detonations are simulated with the realistic heat capacity model and its effects on the detonation featured parameters, such as the detonation velocity and cell width, are analyzed.

The reference [30] provides a numerical investigation of the oblique detonation induced by a finite wedge in hydrogen-air mixtures. The Navier Stocks equations with a chemical model are solved to simulate the flow field characteristic of an oblique detonation wave. The paper summarizes the effects some parameters like the equivalence ratios, expansion waves, the initiation characteristics, wave structures, density, and temperature distributions on the oblique detonation wave.

The reference [29] presents two structures with smooth and abrupt transitions simulated by the twodimensional Euler equations and a two-step induction-reaction model, and their interactions with the cowl-induced expansion wave are investigated.

The aim of this work is to develop a new computational program to study the effect of thermodynamics gas on the $O S W$ and $C S W$ parameters at $H T$ to make a suitable choice of gas which gives good performances toward the shock for aeronautical and aerospace construction, and to solve the future aerodynamics (gas dynamics) problems on the increase of the ambient temperature caused by the increase in holes of the ozone layer, and by the radical and progressive change of the chemical composition of gas of the ambient environment presented by the burning of waste whose volume increases considerably every day, and consequently make applications for various external and internal aerodynamics problems like, the calculation of the suitable intake adaptation parameters, dihedron and cone wave drag, aerodynamic coefficients of a pointed supersonic airfoil and oblique shock reflection. Other interested problem can be solved by the use of this work like the oblique detonation process.

Among the advantages of our elaborate program is that a remarkable improvement on the method of solving conservation equations by the method of Runge Kutta of order 10 which is and important method for the problems for initial condition will be indicated in the paper. While in references $[12,18,19]$ use the finite difference method for the obtained differential equations, which is a rather heavy and slightly precise method. 
TABle 1 . Coefficients of $C_{P}(T)$ and $R$ for some gases.

\begin{tabular}{llllll}
\hline $\mathrm{N}$ & $\mathrm{Gas}$ & $a^{\prime} \mathrm{J} /(\mathrm{K} \mathrm{mol})$ & $b^{\prime} \mathrm{J} /\left(\mathrm{K}^{2} \mathrm{~mol}\right)$ & $c^{\prime} \mathrm{J} \mathrm{K} / \mathrm{mol}$ & $R \mathrm{~J} /(\mathrm{kg} \mathrm{K})$ \\
\hline 1 & $\mathrm{H}_{2}$ & 27.28 & 3.26 & 0.50 & 4157.25 \\
2 & $\mathrm{O}_{2}$ & 29.96 & 4.18 & -1.67 & 259.828 \\
3 & $\mathrm{~N}_{2}$ & 28.58 & 3.76 & -0.50 & 296.946 \\
4 & $\mathrm{CO}$ & 28.41 & 4.10 & -0.46 & 296.946 \\
5 & $\mathrm{CO}_{2}$ & 44.22 & 8.79 & -8.62 & 188.965 \\
6 & $\mathrm{H}_{2} \mathrm{O}$ & 30.54 & 10.29 & 0.08 & 461.916 \\
7 & $\mathrm{NH}_{3}$ & 29.75 & 25.10 & -1.55 & 489.088 \\
8 & $\mathrm{CH}_{4}$ & 23.64 & 47.86 & -1.92 & 519.656 \\
9 & Air & Polynomial of 9th degree [5, 7-9, 12,31] & 287.102 \\
\hline
\end{tabular}

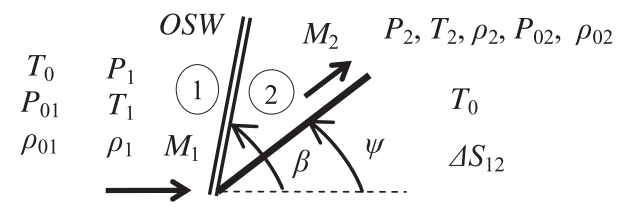

Figure 1. OSW illustration.

At high $T_{0}, M_{1}$ and $\varphi$ applications which exceed respectively $240 \mathrm{~K}, 2.00$ and $10^{\circ}$, the $P G$ model do not gives good results and a correction is necessary to this model by developing a new and adequate model called by $H T$ model for this application, which answers the behavior of the flow in this case. The problem occurs in the energy equation, where $C_{P}(T)$ depends on the temperature, lower than threshold dissociation of the molecules $[12,16,17,31]$ which is not the case for the $P G$ model, where $C_{P}$ is considered as constant $[1-3,22,24,25]$. The shock is characterized by the determination of the thermodynamic parameters and the Mach number through the shock as well as the increase of the entropy.

Among several gases existed in nature, The $\mathrm{H}_{2}, \mathrm{O}_{2}, \mathrm{~N}_{2}, \mathrm{CO}, \mathrm{CO}_{2}, \mathrm{H}_{2} \mathrm{O}, \mathrm{NH}_{3}, \mathrm{CH}_{4}$ and air are chosen in this study $[4-6,8-10,14,20,21]$. The gas is considered as perfect. Including the state equation $(P=\rho R T)$ is still valid. It is considered calorically imperfect seen the change in $C_{P}(T)$ varies with temperature and thermally perfect because the dissociation of molecules is not reached. For the chosen gas is found in a variation according to the relation (1.1) of $C_{P}(T)$. The coefficients of this function and the constant $R$ of the gas are shown in Table $1[4,6,8-10,14,20,21,23]$.

$$
C_{P}(T)=a^{\prime}+b^{\prime} T+\frac{c^{\prime}}{T^{2}}
$$

Concerning the air, the variation of $C_{P}(T)$ is chosen as a polynomial of 9 th degree $[4,5,7-9,12]$.

The function $C_{P}(T)$ is found in Joule/(mol K). One needs to convert it to the Joule/( $\left.\mathrm{kg} \mathrm{K}\right)$. For this we used the relationship (1.2).

$$
C_{P}\left(\frac{\text { Joule }}{\operatorname{kg~K}}\right)=C_{P}\left(\frac{\text { Joule }}{\mathrm{mol} \mathrm{K}}\right) \times \frac{R}{8.3145},
$$

\section{2. $O S W$ FORMULATION AT $H T$}

Figure 1 represents a general diagram of an $O S W$ on a dihedron inclined by an angle $\psi$ and the envisaged parameters. 
The determination of the parameters $\left(M_{2}, \beta, T_{2} / T_{1}, \rho_{2} / \rho_{1}\right.$ and $\left.P_{2} / P_{1}\right)$ by $(2.1),(2.2),(2.3)$ and $(2.4)$ through the $O S W[16,17]$, in the first step, The $H T$ model presented in $[12,16,17,27]$ has been improved after making a correction to the relation between $\beta, \psi$ and $M_{1}$, since the authors in these references have used the equation designed for the $P G$ model, at constant $C_{P}[1,3]$, given the difficulty of finding an analytic form, which gives results far from reality, and which does not respond to the behavior of $H T$ flow. The said equation is the most interesting in the calculation of the $O S W$ parameters, since all the other parameters depend on $\beta$, $\psi$ and $M_{1}$.

The relationships are summarized as follows $[16,17,27]$ :

$$
\begin{gathered}
M_{2}^{2}=\frac{1-\frac{P_{2}}{P_{1}}}{\sin ^{2}(\beta-\psi) \times \gamma\left(T_{2}\right) \times\left[1-\frac{\rho_{2}}{\rho_{1}}\right] \frac{P_{2}}{P_{1}}}, \\
\frac{T_{2}}{T_{1}}=\frac{\frac{\rho_{2}}{\rho_{1}}-M_{1}^{2} \sin ^{2}(\beta) \times \gamma\left(T_{1}\right) \times\left(1-\frac{\rho_{2}}{\rho_{1}}\right)}{\left(\frac{\rho_{2}}{\rho_{1}}\right)^{2}}, \\
\frac{\rho_{2}}{\rho_{1}}=\frac{\tan (\beta)}{\tan (\beta-\psi)}, \\
\frac{P_{2}}{P_{1}}=\left[\frac{\rho_{2}}{\rho_{1}}\right]\left[\frac{T_{2}}{T_{1}}\right] .
\end{gathered}
$$

Two solutions can be found depending on the value of the obtained $M_{2}$, which implies that all the physical parameters will admit two solutions. If $M_{2} \geq 1$, we get a weak shock. If $M_{2}<1$, we will have a strong shock. In general, the weak shock that occurs in nature $[2,3,22,25]$.

Before the determination of the parameters through the $O S W$, it is necessary to determine, in the first place, the angle $\beta$ corresponding to $\psi$ and $M_{1}$ and the thermodynamics of the used gas. In this work, we will determine the $\beta$ deviation with high accuracy according to the real $H T$ model by applying the principle of $H T N S W$ [31].

By varying $M_{1}, T_{0}, \psi$ and the used gas, one can determine $\beta, M_{2}, T_{2} / T_{1}, P_{2} / P_{1}$ and $\rho_{2} / \rho_{1}$ through an $O S W$.

\section{3. $C S W$ FORMULATION AT $H T$}

Figure 2 represents a general diagram of a $C S W$ and the physical parameters presented as well as the adaptation of the cylindrical coordinates.

First, the parameters must be determined just after the shock by the numerical resolution of the equations (2.1), (2.2), (2.3) of $O S W$ [12]. The second step consists in finding the parameters of the isentropic flow in the region just after the shock and the cone surface corresponding to $\theta C$, by numerical resolution of the differential equations (3.1), (3.2) and (3.3) coupled to three variables ( $V r, V \theta$ and $T$ ) simultaneously by the adaptation of the Runge Kutta method of order $10[11,15,23]$ with initial condition. As a result, all other parameters $M_{C}$, $T_{C} / T_{0}, \rho_{C} / \rho_{02}$ and $P_{C} / P_{02}$ on the cone surface will be determined. In this case, the isentropic parameters between the shock and the cone surface depend on the angle $\theta$.

$$
\frac{\mathrm{d} V_{r}}{\mathrm{~d} \theta}=V_{\theta},
$$




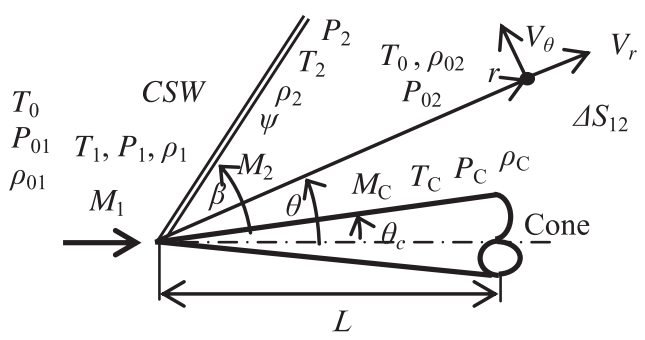

Figure 2. CSW illustration.

$$
\begin{gathered}
\frac{\mathrm{d} V_{\theta}}{\mathrm{d} \theta}=\frac{a(T)\left[2 V_{r} \tan (\theta)+V_{\theta}\right]-V_{\theta}^{2} V_{r} \tan (\theta)}{\tan (\theta)\left[V_{\theta}^{2}-a^{2}(T)\right]}, \\
\frac{\mathrm{d} T}{\mathrm{~d} \theta}=\frac{V_{\theta} a(T)\left[V_{r} \tan (\theta)+V_{\theta}\right]}{\tan (\theta) C_{P}(T)\left[a^{2}(T)-V_{\theta}^{2}\right]}, \\
V^{2}=V_{r}^{2}+V_{\theta}^{2}, \\
V=\frac{M}{a},
\end{gathered}
$$

where

$$
\begin{aligned}
& a(T)=\sqrt{\gamma(T) R T}, \\
& \gamma(T)=\frac{C_{P}(T)}{C_{P}(T)-R} .
\end{aligned}
$$

By varying $M_{1}, T_{0}, \theta_{C}$ and the gas used, we can find all the parameters $\beta, \psi, M_{2}, T_{2} / T_{1}, \rho_{2} / \rho 1$ and $P_{2} / P_{1}$ through a $C S W$.

\section{SHOCK INTENSITY AND ENTROPY JUMP}

The total pressures ratio and the entropy jump through the $O S W$ or $C S W$ can be determined respectively by the following relation $[2,3]$ :

$$
\begin{gathered}
\frac{P_{0_{2}}}{P_{0_{1}}}=\left[\frac{P_{2}}{P_{1}}\right]\left[\frac{P_{1}}{P_{0_{1}}}\right] /\left[\frac{P_{2}}{P_{0_{2}}}\right], \\
\frac{\Delta S_{12}}{R}=-\log \left(\frac{P_{0_{2}}}{P_{0_{1}}}\right) .
\end{gathered}
$$

We notice that $T_{02}=T_{01}=T_{0}, \rho_{02} \equiv \rho_{01}$ and $P_{02} \equiv P_{01}$. 


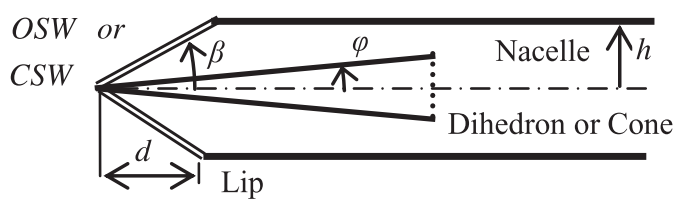

FiguRE 3. Distance for an adapted intake.

\section{ISENTROPIC PARAMETERS}

The isentropic values [32] can therefore be determined immediately after the shock and on the cone surface by the relations (5.1), (5.2) and (5.3) respectively when $\left(M=M_{2}, T=T_{2}, \rho_{0}=\rho_{02}, P_{0}=P_{02}\right)$ or $\left(M=, M_{C}\right.$, $\left.T=T_{C}, \rho_{0}=\rho_{02}, P_{0}=P_{02}\right)$.

$$
\begin{gathered}
M(T)=\frac{\sqrt{2 H(T)}}{a(T)}, \\
\frac{\rho}{\rho_{0}}=\operatorname{Exp}\left(-\int_{T}^{T_{0}} \frac{C P(T)}{a^{2}(T)} \mathrm{d} T\right), \\
\frac{P}{P_{0}}=\left[\frac{T}{T_{0}}\right]\left[\frac{\rho}{\rho_{0}}\right],
\end{gathered}
$$

With $[23]$

$$
H(T)=\int_{T}^{T_{0}} C_{P}(T) \mathrm{d} T
$$

\section{Applications}

The flow out of an adaptation in a supersonic nozzle is a good example for $O S W$ application. When $P_{E}<P_{a}$, $O S W$ will be to occur in the interior of the nozzle, and $M E$ will no longer supersonic, and necessarily affects the nozzle $C_{F}$.

The determination of $\lambda$ of a supersonic aircraft intake engine is a good example for $O S W$ and $C S W$ applications.

Figure 3 shows a diagram of a suitable air intake. The initial position of the dihedral or central cone is given when its leading edge is on the same vertical line as the retraction of the nacelle.

In order for the air intake to be adapted, for not having the reflection of the shock wave inside the engine, the distance of the displacement of the dihedral or the central cone must be calculated by the following relation:

$$
\lambda=\frac{\mathrm{d}}{h}=\frac{1}{\tan (\beta)} .
$$

The value of $\beta$ depends on $M_{1}, T_{0}, \varphi$, the gas used and the dihedron or cone application.

If the distance $\lambda$ given by (6.1) is not respected, the shock wave propagates inside the motor and consequently there will be a considerable increase in the drag force, which gives the adaptation of the intake is needed to decrease the drag and to have favorite's parameters after the shock. 


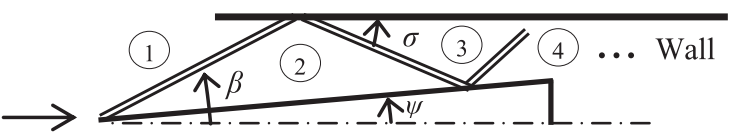

FiguRE 4. Shock wave reflection in an unsuitable air intake.

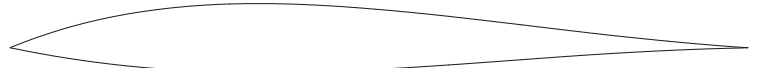

FiguRE 5. Supersonic pointed curved non symmetrical airfoil.

In the case where the air intake is not suitable, we will therefore have the configuration shown in Figure 4 according to the lip shape of the nacelle with a weak $O S W$ reflection. The parameters in the zone 3 can be calculated in the same way as the calculation in the zone 2 after the determination of $\beta_{3}$ and $M_{3}$ in the zone 3 . The shock wave will be reflected by an angle $\sigma$ with respect to the horizontal wall as shown in Figure 4. However $\sigma=\beta_{3}-\psi$. Continuous reflection will be observed up to the motor exit. Then to avoid the reflection of the shock wave, the central body of the air intake must be placed according to Figure 4. Hence the computation of $\lambda$ by (6.1) is necessary.

The calculation of the wave drag force exerted on the dihedral or cone surface plays a very important role in the aeronautical applications. It can be shown what is given by the normalized formula (6.2). Knowing that $\left(k=1, \varphi=\psi, \omega=2, P=P_{2}\right)$ for the dihedral and $\left(k=2, \varphi=\theta_{C}, \omega=\pi, P=P_{C}\right)$ for the cone.

$$
D=\frac{\operatorname{Drag}}{\operatorname{Ll} P_{0}}=\omega \tan ^{K}(\varphi)\left(\frac{P}{P_{0_{2}}}\right)\left(\frac{P_{0_{2}}}{P_{0_{1}}}\right)
$$

The values of $P / P_{02}$ and $P_{02} / P_{01}$ in (6.2) are respectively given by (5.3) and (4.1) respectively when $T=T_{2}$, $\rho=\rho_{2}$ and $P=P_{2}, \rho_{0}=\rho_{02}$ and $P=P_{02}$ for the dihedron, and when $T=T_{C}, \rho=\rho_{C}$ and $P=P_{C}, \rho_{0}=\rho_{02}$ for the cone. While $\varphi$ and $P_{0}$ are among the data of our study. We have $P_{0}=P_{01}$.

The $C_{x}, C_{y}$ and $C_{m}$, and supersonic flow calculation for a given pointed airfoil is a fourth example for our applications. In Figure 5, a non-symmetrical curved pointed airfoil has been presented in an $H T$ supersonic flow and under $\alpha, M_{1}$ and $T_{0}$ data. The extrados and the intrados airfoil equations $f_{E}(x)$ and $f_{I}(x)$ are given by the relations (6.3) and (6.4).

$$
\begin{aligned}
& f_{E}(x)=\frac{x}{10 C}\left(1-\frac{x}{C}\right)\left(1-\frac{2 x}{3 C}\right)\left(1-\frac{x}{2 C}\right), \\
& f_{I}(x)=-\frac{x}{20 C}\left(1-\frac{x}{C}\right)\left(1-\frac{3 x}{4 C}\right)\left(1-\frac{5 x}{8 C}\right) .
\end{aligned}
$$

Where $0=x / C=1$.

\section{RESUlts AND COMMENTS}

The presentation of the results for each shock wave is preferred in separate figures for good illustration.

All figures contain 9 curves for 9 different selected gases. Curve 1 for the $\mathrm{H}_{2}$ gas. Curve 2 for $\mathrm{O}_{2}$. Curve 3 for $\mathrm{N}_{2}$. Curve 4 for CO. Curve $5 \mathrm{CO}_{2}$. Curve 6 for $\mathrm{H}_{2} \mathrm{O}$. Curve 7 for $\mathrm{NH}_{3}$. Curve 8 for $\mathrm{CH}_{4}$ and the curve 9 for air.

The choice of 9 gases in this study is based to have 3 or 5 gases giving better choices compared to air, and 5 or 3 other gases giving a bad choice compared to air. 
TABLE 2. Gas effect on $\psi_{\max }$ for $O S W$ at $T_{0}=2000 \mathrm{~K}$.

\begin{tabular}{llllll}
\hline $\mathrm{N}$ & Gas & $M_{1}=2.00$ & $M_{1}=3.00$ & $M_{1}=4.00$ & $M_{1}=5.00$ \\
\hline 1 & $\mathrm{H}_{2}$ & 24.0451 & 35.5024 & 40.1863 & 42.4514 \\
2 & $\mathrm{O}_{2}$ & 24.9934 & 37.1373 & 42.1355 & 44.5402 \\
3 & $\mathrm{~N}_{2}$ & 24.5553 & 36.3724 & 41.2163 & 43.5503 \\
4 & $\mathrm{CO}$ & 24.6511 & 36.5123 & 41.3614 & 43.6912 \\
5 & $\mathrm{CO}_{2}$ & 27.7378 & 42.1727 & 48.4294 & 51.5240 \\
6 & $\mathrm{H}_{2} \mathrm{O}$ & 26.7619 & 40.1466 & 45.6516 & 48.2529 \\
7 & $\mathrm{NH}_{3}$ & 28.7320 & 43.8759 & 50.3417 & 53.3940 \\
8 & $\mathrm{CH}_{4}$ & 29.8985 & 46.2645 & 53.6026 & 57.2007 \\
9 & Air & 24.6714 & 36.7154 & 41.0122 & 43.8995 \\
\hline
\end{tabular}

TABLE 3. Gas effect on $\psi_{\max }$ for $O S W$ when $M_{1}=3.00$.

\begin{tabular}{llllll}
\hline $\mathrm{N}$ & Gas & $T_{0}=298$ & $T_{0}=1000 \mathrm{~K}$ & $T_{0}=2000 \mathrm{~K}$ & $T_{0}=3000 \mathrm{~K}$ \\
\hline 1 & $\mathrm{H}_{2}$ & 34.0020 & 34.3722 & 35.5024 & 36.5497 \\
2 & $\mathrm{O}_{2}$ & 34.1182 & 35.8017 & 37.1373 & 38.2496 \\
3 & $\mathrm{~N}_{2}$ & 33.0729 & 35.1175 & 36.3724 & 37.4687 \\
4 & $\mathrm{CO}$ & 33.7975 & 35.1575 & 36.5123 & 37.6854 \\
5 & $\mathrm{CO}_{2}$ & 38.6914 & 40.7564 & 42.1727 & 43.1916 \\
6 & $\mathrm{H}_{2} \mathrm{O}$ & 35.8732 & 37.8834 & 40.1466 & 41.8194 \\
7 & $\mathrm{NH}_{3}$ & 38.1136 & 40.7382 & 43.8759 & 45.6144 \\
8 & $\mathrm{CH}_{4}$ & 39.8452 & 42.9084 & 46.2645 & 47.7252 \\
9 & Air & 33.9774 & 35.1931 & 36.7154 & 37.7759 \\
\hline
\end{tabular}

In the figures of $O S W$ and $C S W$, we presented the variation of all parameters only for the case of the $W S$, except the parameters $\beta, \psi, M_{2}$ and $M_{C}$, where we added the variation for the $S S$.

The commentary of the results was left at the end of the presentation of all $O S W$ and $C S W$ figures to avoid repetition of comments.

It may even be noted in all the presented figures that the arrangement of the numbers of the 9 curves of the selected gases for maximum (or minimum) to minimum (or maximum) value, in each figure and tables, is the same for all parameters and for all $O S W$ and $C S W$. It is given by this order 1, 3, 4, 9, 2, 6, 5, 7 and 8 .

For comparison, the results for air (curve 9) of $H T$ model can be found in $[2,3,13,16,17,22,25-27]$ for $O S W$ and in [12] for $C S W$. It should be noted that the $H T$ model presented in these references is different from the one presented in this study according to the modifications discussed in the introduction.

For $P G$ model, the results can be found in [1] for $N S W$ and $O S W$ and in [18, 19,24] for $C S W$ also for comparison.

\subsection{Results for $O S W$}

Table 2 shows the gas and $M_{1}$ effect on the $\psi_{M a x}$ to have an attached $O S W$ when $T_{0}=2000 \mathrm{~K}$.

Table 3 shows the gas and $T_{0}$ effect on the $\psi_{\text {Max }}$ to have an attached $O S W$ when $M_{1}=3.00$.

Table 4 shows the gas and $\psi$ effect on the minimum value of $M_{1}$ to have an attached $O S W$ when $T_{0}=2000$ K.

Table 5 shows the gas and $T_{0}$ effect on the minimum value of $M_{1}$ to have an attached $O S W$ when $\psi=20^{\circ}$.

Table 6 shows an example of gas effect on the aerodynamic coefficients of the airfoil of Figure 5 only for weak $O S W$ when $M_{1}=3.00, T_{0}=2000 \mathrm{~K}$ and $\alpha=4^{\circ}$.

Table 7 represents an example of calculation of $M, \sigma$ and $\beta$ in the different isentropic flow zones after the reflection of a Weak $O S W$ when $M_{1}=3.00, T_{0}=2000 \mathrm{~K}$ and $\psi=10^{\circ}$. Other parameters such as $T_{2} / T_{1}, \rho_{2} / \rho_{1}$, 
TABLE 4. Gas effect on $M_{L i m}$ for $O S W$ at $T_{0}=2000 \mathrm{~K}$.

\begin{tabular}{llllll}
\hline $\mathrm{N}$ & Gas & $\psi=10^{\circ}$ & $\psi=20^{\circ}$ & $\psi=30^{\circ}$ & $\psi=40^{\circ}$ \\
\hline 1 & $\mathrm{H}_{2}$ & 1.4055 & 1.7964 & 2.4015 & 3.9413 \\
2 & $\mathrm{O}_{2}$ & 1.3953 & 1.7645 & 2.3057 & 3.4736 \\
3 & $\mathrm{~N}_{2}$ & 1.3999 & 1.7787 & 2.3476 & 3.6659 \\
4 & $\mathrm{CO}$ & 1.3987 & 1.7754 & 2.3387 & 3.6310 \\
5 & $\mathrm{CO}_{2}$ & 1.3705 & 1.6915 & 2.1083 & 2.7808 \\
6 & $\mathrm{H}_{2} \mathrm{O}$ & 1.3780 & 1.7140 & 2.1691 & 2.9822 \\
7 & $\mathrm{NH}_{3}$ & 1.3624 & 1.6697 & 2.0566 & 2.6484 \\
8 & $\mathrm{CH}_{4}$ & 1.3544 & 1.6477 & 2.0041 & 2.5133 \\
9 & Air & 1.3975 & 1.7751 & 2.3325 & 3.5731 \\
\hline
\end{tabular}

TABLE 5. Gas effect on $M_{l i m}$ for $O S W$ when $\psi=20^{\circ}$.

\begin{tabular}{llllll}
\hline $\mathrm{N}$ & Gas & $T_{0}=298$ & $T_{0}=1000 \mathrm{~K}$ & $T_{0}=2000 \mathrm{~K}$ & $T_{0}=3000 \mathrm{~K}$ \\
\hline 1 & $\mathrm{H}_{2}$ & 1.8451 & 1.8264 & 1.7964 & 1.7729 \\
2 & $\mathrm{O}_{2}$ & 1.8398 & 1.7932 & 1.7645 & 1.7435 \\
3 & $\mathrm{~N}_{2}$ & 1.8407 & 1.8083 & 1.7787 & 1.7563 \\
4 & $\mathrm{CO}$ & 1.8417 & 1.8070 & 1.7754 & 1.7521 \\
5 & $\mathrm{CO}_{2}$ & 1.7502 & 1.7104 & 1.6915 & 1.6792 \\
6 & $\mathrm{H}_{2} \mathrm{O}$ & 1.7948 & 1.7510 & 1.7140 & 1.6922 \\
7 & $\mathrm{NH}_{3}$ & 1.7462 & 1.7053 & 1.6697 & 1.6534 \\
8 & $\mathrm{CH}_{4}$ & 1.7182 & 1.6785 & 1.6477 & 1.6360 \\
9 & Air & 1.8404 & 1.8028 & 1.7759 & 1.7507 \\
\hline
\end{tabular}

TABle 6. Gas effect on $C_{x}, C_{y}, C_{m}$ and $\Delta S / R$ for the airfoil of Figure 5 when $M_{1}=3.00$, $T_{0}=2000 \mathrm{~K}$ and $\alpha=4^{\circ}$.

\begin{tabular}{lllllll}
\hline $\mathrm{N}$ & Gas & $C_{x} \times 10^{4}$ & $C_{y} \times 10^{2}$ & $C_{m} \times 10^{2}$ & $\Delta S / R \times 10$ & $\chi$ \\
\hline 1 & $\mathrm{H}_{2}$ & 0.2928 & 0.2640 & 0.1340 & 0.1204 & 90.163 \\
2 & $\mathrm{O}_{2}$ & 0.2766 & 0.2490 & 0.1266 & 0.1137 & 90.021 \\
3 & $\mathrm{~N}_{2}$ & 0.2844 & 0.2562 & 0.1302 & 0.1166 & 90.091 \\
4 & $\mathrm{CO}$ & 0.2835 & 0.2554 & 0.1297 & 0.1155 & 90.088 \\
5 & $\mathrm{CO}_{2}$ & 0.2216 & 0.1986 & 0.1013 & 0.1005 & 89.620 \\
6 & $\mathrm{H}_{2} \mathrm{O}$ & 0.2472 & 0.2219 & 0.1130 & 0.1009 & 89.765 \\
7 & $\mathrm{NH}_{3}$ & 0.2051 & 0.1836 & 0.0936 & 0.0927 & 89.517 \\
8 & $\mathrm{CH}_{4}$ & 0.1773 & 0.1585 & 0.0809 & 0.0906 & 89.396 \\
9 & Air & 0.2781 & 0.2505 & 0.1273 & 0.1151 & 90.075 \\
\hline
\end{tabular}

$P_{2} / P_{1}, P_{02} / P_{01}, \Delta S 12 / R$ between zone 2 and 1 and $T_{2} / T_{0}, \rho_{2} / \rho_{02}, P_{2} / P_{02}$ in zone 2 , as well as $T_{3} / T_{2}, \rho_{3} / \rho_{2}$, $P_{3} / P_{2}, P_{03} / P_{02}, \Delta S 23 / R$ between zones 3 and 2 , and $T_{3} / T_{0}, \rho_{3} / \rho_{03}, P_{3} / P_{03}$ in zone 3 , and $T_{3} / T_{1}, \rho_{3} / \rho_{1}$, $P_{3} / P_{1}, P_{03} / P_{01}, \Delta S_{13} / R$ between zones 1 and $T_{4} / T_{3}, \rho_{4} / \rho_{3}, P 4 / P_{3}, P_{0} 4 / P_{03}, \Delta S 34 / R$ between zones 3 and 4 , and $T_{4} / T_{0}, \rho_{4} / \rho_{0} 4, P 4 / P_{0} 4$ in the zone 4 , and $T_{4} / T_{1}, \rho_{4} / \rho_{1}, P 4 / P_{1}, P_{0} 4 / P_{01}, \Delta S_{14} / R$ between zones 1 and 4 can be easily deduced directly according to the values of Table 7 .

Figure 6 shows the gas effect on the variation of $\beta, M_{2}, T_{2} / T_{1}, \rho_{2} / \rho_{1}, P_{2} / P_{1}, P_{02} / P_{01}$ and $\Delta S 12 / R$ through a weak $O S W$ versus of $\psi$ for $M_{1}=3.00$ and $T_{0}=2000 \mathrm{~K}$. Only for $\beta$ and $M_{2}$ was added the variation for the strong shock.

Figure 7 shows the effect of gas on the variation of the isentropic parameters $T_{2} / T_{0}, \rho_{2} / \rho_{02}$ and $P_{2} / P_{02}$ after weak $O S W$ versus $\psi$ when $M_{1}=3.00$ and $T_{0}=2000 \mathrm{~K}$. 


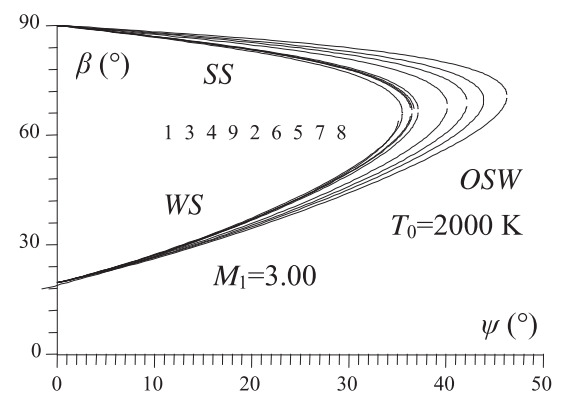

(a) : Variation of $\beta$ versus $\psi$.

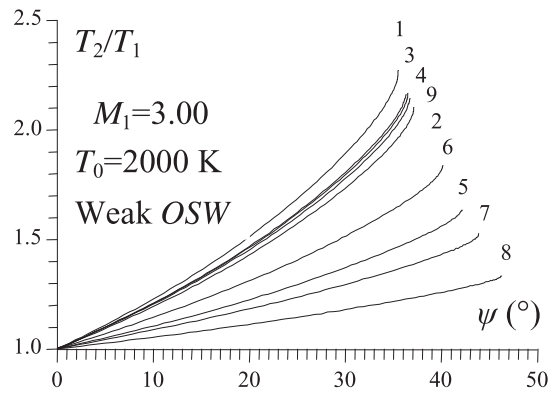

(c) : Variation of $T_{2} / T_{1}$ versus $\psi$.

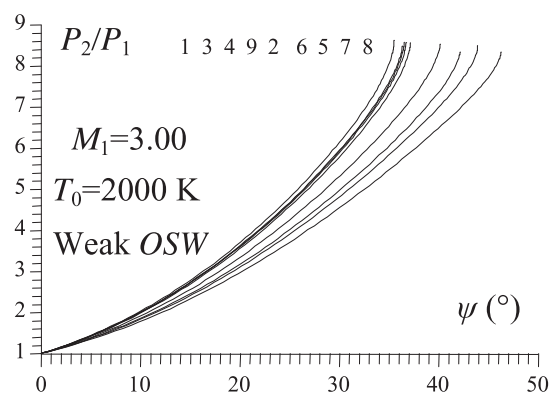

(e) : Variation of $P_{2} / P_{1}$ versus $\psi$.

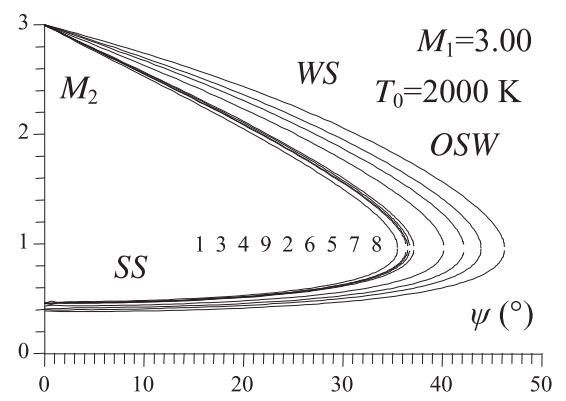

(b) : Variation of $M_{2}$ versus $\psi$.

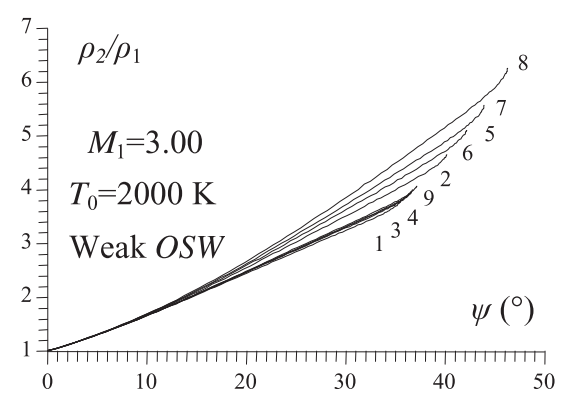

(d) : Variation of $\rho_{2} / \rho_{1}$ versus $\psi$.

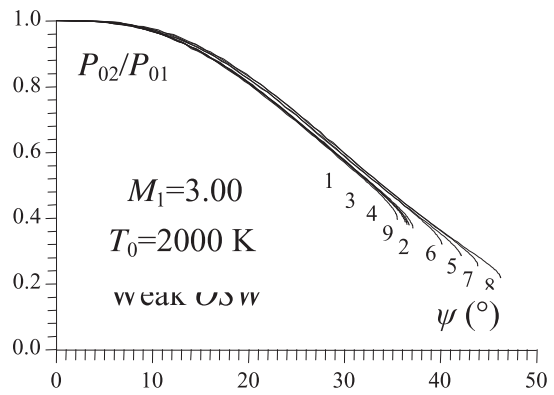

(f) : Variation of $P_{02} / P_{01}$ versus $\psi$.

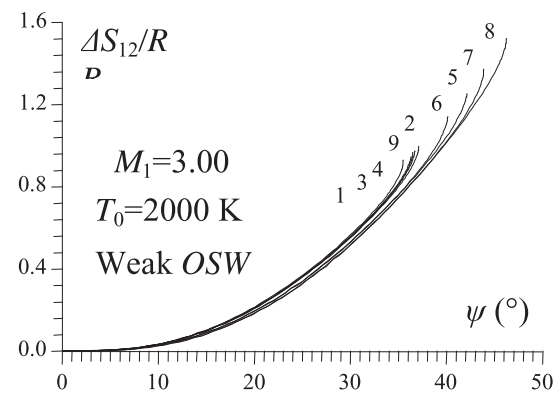

(g) : Variation of $\Delta S_{12} / R$ versus $\psi$.

Figure 6. Gas effect on the variation of $O S W$ parameters versus $\psi$. 
TABLE 7. Gas effect on the $O S W$ reflection parameters of figure 4 when $M_{1}=3.00, \psi=10^{\circ}$ and $T_{0}=2000 \mathrm{~K}$.

\begin{tabular}{llllllll}
\hline & \multicolumn{3}{c}{ Zone 2 } & \multicolumn{2}{c}{ Zone 3 } & \multicolumn{2}{c}{ Zone 4} \\
\hline $\mathrm{N}$ & Gas & $\beta 2\left(^{\circ}\right)$ & $M_{2}$ & $\beta 3\left(^{\circ}\right)$ & $M 3$ & $\beta 4\left(^{\circ}\right)$ & $M 4$ \\
\hline 1 & $\mathrm{H}_{2}$ & 27.230 & 2.528 & 31.340 & 2.133 & 36.600 & 1.784 \\
2 & $\mathrm{O}_{2}$ & 27.016 & 2.566 & 30.720 & 2.194 & 35.385 & 1.861 \\
3 & $\mathrm{~N}_{2}$ & 27.114 & 2.549 & 31.003 & 2.166 & 35.935 & 1.825 \\
4 & $\mathrm{CO}$ & 27.099 & 2.551 & 30.959 & 2.170 & 35.845 & 1.831 \\
5 & $\mathrm{CO}_{2}$ & 26.474 & 2.669 & 29.158 & 2.366 & 32.433 & 2.082 \\
6 & $\mathrm{H}_{2} \mathrm{O}$ & 26.690 & 2.626 & 29.781 & 2.294 & 33.571 & 1.991 \\
7 & $\mathrm{NH}_{3}$ & 26.340 & 2.695 & 28.779 & 2.412 & 31.714 & 2.144 \\
8 & $\mathrm{CH}_{4}$ & 26.148 & 2.735 & 28.229 & 2.482 & 30.718 & 2.238 \\
9 & Air & 27.059 & 2.559 & 30.837 & 2.183 & 35.620 & 1.845 \\
\hline
\end{tabular}

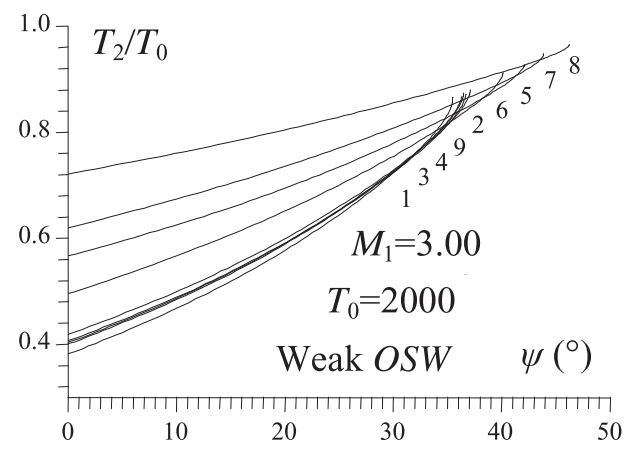

(a) : Variation of $T_{2} / T_{0}$ versus $\psi$.

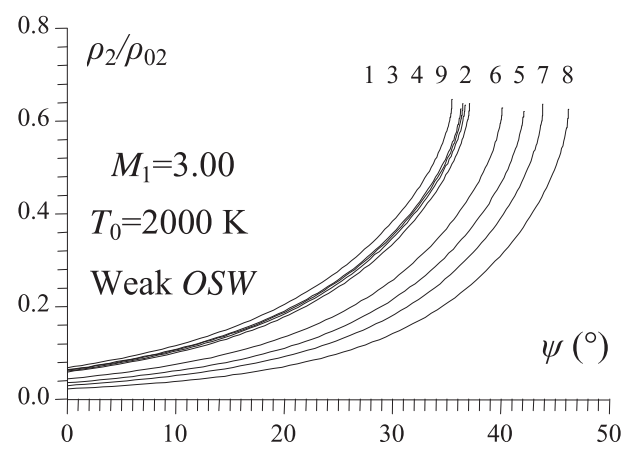

(b) : Variation of $\rho_{2} / \rho_{02}$ versus $\psi$.

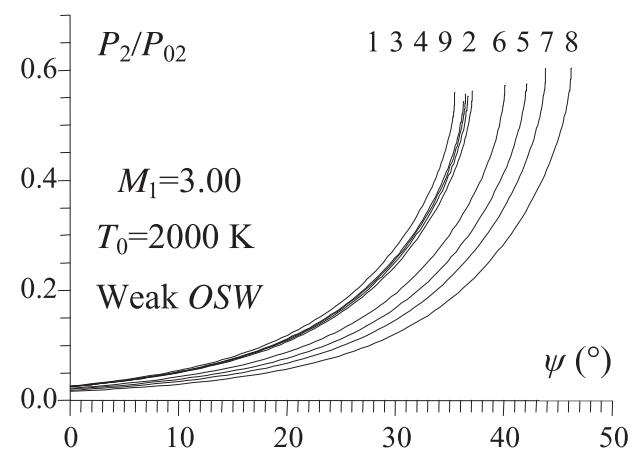

(c) : Variation of $P_{2} / P_{02}$ versus $\psi$.

Figure 7. Gas effect on the variation of the isentropic parameters behind $O S W$ versus $\psi$.

Figure 8 shows the gas effect on the variation of the adaptation distance $\lambda$ of a $2 \mathrm{D}$ air intake as a function of $\psi$ for weak $O S W$ when $M_{1}=3.00$ and $T_{0}=2000 \mathrm{~K}$.

Figure 9 shows the gas effect on the variation of the wave $\operatorname{drag} D$ on the dihedral as a function of $\psi$ for weak $O S W$ when $M_{1}=3.00$ and $T_{0}=2000 \mathrm{~K}$.

Figure 10 shows the gas effect on the variation of $\beta, M_{2}, T_{2} / T_{1}, \rho_{2} / \rho_{1}, P_{2} / P_{1}, P_{02} / P_{01}$ and $\Delta S 12 / R$ through a weak $O S W$ versus of $M_{1}$ for $\psi=20^{\circ}$ and $T_{0}=2000 \mathrm{~K}$. Only for $\beta$ and $M_{2}$ was added the variation for the strong shock. 


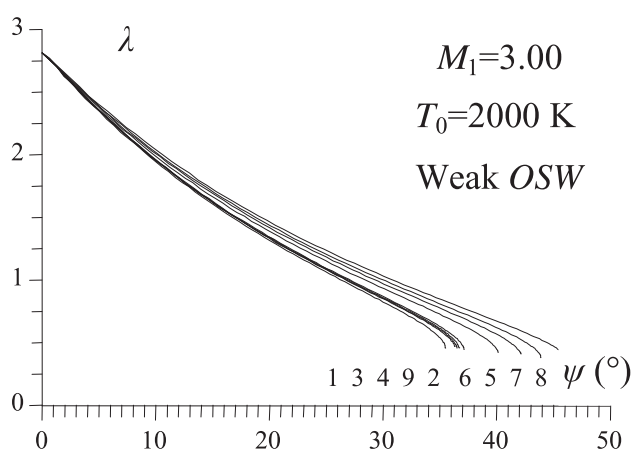

Figure 8. Gas effect on the variation of $\lambda$ versus $\psi$.

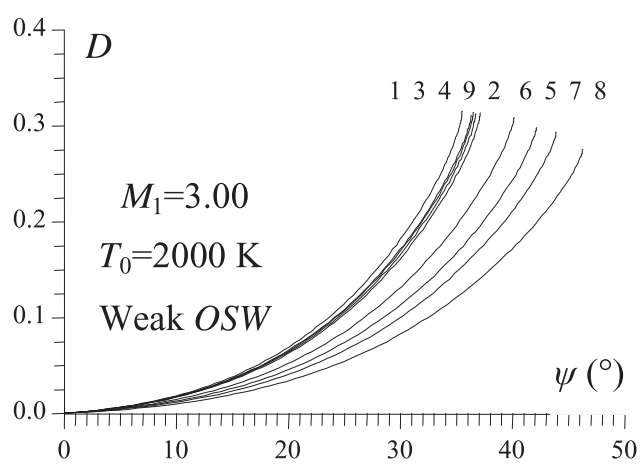

FiguRE 9. Gas effect on the variation of $D$ versus $\psi$.

Figure 11 shows the effect of gas on the variation of the isentropic parameters $T_{2} / T_{0}, \rho_{2} / \rho_{02}$ and $P_{2} / P_{02}$ after weak $O S W$ versus $M_{1}$ when $\psi=20^{\circ}$ and $T_{0}=2000 \mathrm{~K}$.

Figure 12 shows the gas effect on the variation of the adaptation distance $\lambda$ of a $2 \mathrm{D}$ air intake as a function of $M_{1}$ for weak $O S W$ when $\psi=20^{\circ}$ and $T_{0}=2000 \mathrm{~K}$.

Figure 13 shows the gas effect on the variation of the wave drag $D$ on the dihedral as a function of $M_{1}$ for weak $O S W$ when $\psi=20^{\circ}$ and $T_{0}=2000 \mathrm{~K}$.

\subsection{Results for $C S W$}

Table 8 shows the gas and $M_{1}$ effect on the $\psi M a x$ to have an attached $C S W$ when $T_{0}=2000 \mathrm{~K}$.

Table 9 shows the gas and $T_{0}$ effect on the $\psi M a x$ to have an attached $C S W$ when $M_{1}=3.00$.

Table 10 shows the gas and $\psi$ effect on the minimum value of $M_{1}$ to have an attached $C S W$ when $T_{0}=2000$ $\mathrm{K}$.

Table 5 shows the gas and $T_{0}$ effect on the minimum value of $M_{1}$ to have an attached $C S W$ when $\psi=20^{\circ}$.

Figure 14 shows the gas effect on the variation of $\beta, \psi, M_{2}, T_{2} / T_{1}, \rho_{2} / \rho_{1}, P_{2} / P_{1}, P_{02} / P_{01}$ and $\Delta S 12 / R$ through a weak $C S W$ versus of $\theta C$ for $M_{1}=3.00$ and $T_{0}=2000 \mathrm{~K}$. Only for $\beta \psi$ and $M_{2}$ was added the variation for the strong shock.

Figure 15 shows the effect of gas on the variation of the isentropic parameters $T_{2} / T_{0}, \rho_{2} / \rho_{02}$ and $P_{2} / P_{02}$ after weak $C S W$ versus $\theta C$ when $M_{1}=3.00$ and $T_{0}=2000 \mathrm{~K}$.

Figure 16 shows the effect of gas on the variation of the isentropic parameters $M_{C}, T_{C} / T_{0}, \rho_{C} / \rho_{02}$ and $P_{C} / P_{02}$ on the cone surface for weak $C S W$ versus $\theta C$ when $M_{1}=3.00$ and $T_{0}=2000 \mathrm{~K}$. Only for $M_{C}$ was added the variation for strong shock. 


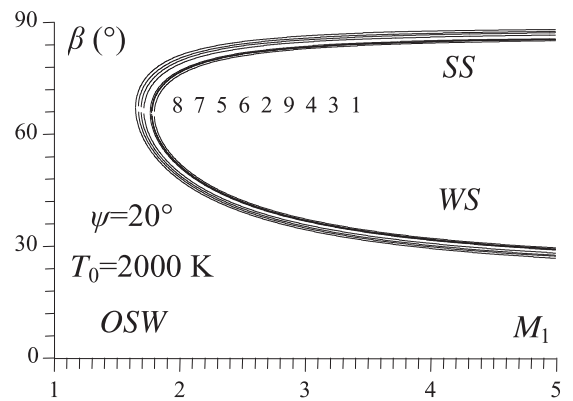

(a) : Variation of $\beta$ versus $M_{1}$.

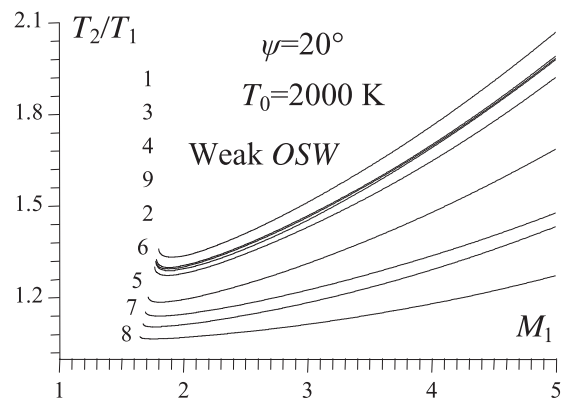

(c) : Variation of $T_{2} / T_{1}$ versus $M_{1}$.

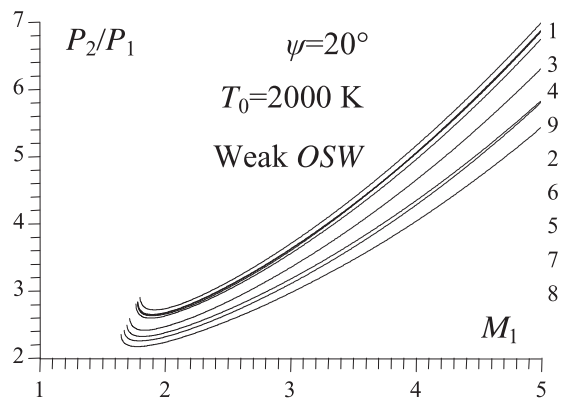

(e) : Variation of $P_{2} / P_{1}$ versus $M_{1}$.

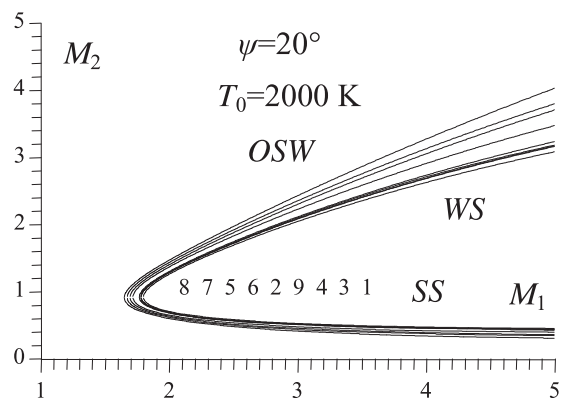

(b) : Variation of $M_{2}$ versus $M_{1}$.

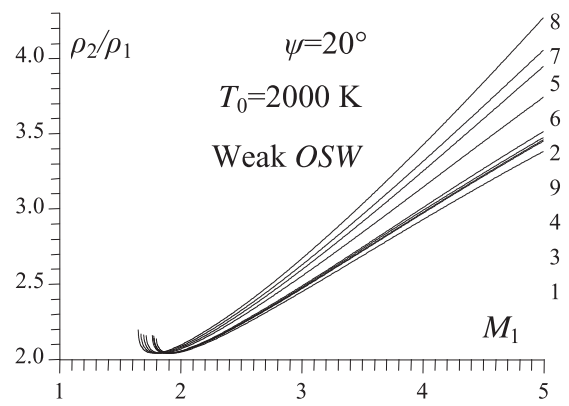

(d) : Variation of $\rho_{2} / \rho_{1}$ versus $M_{1}$.

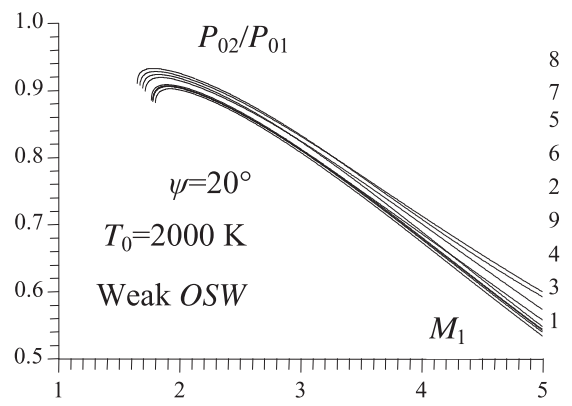

(f) : Variation of $P_{02} / P_{01}$ versus $M_{1}$.

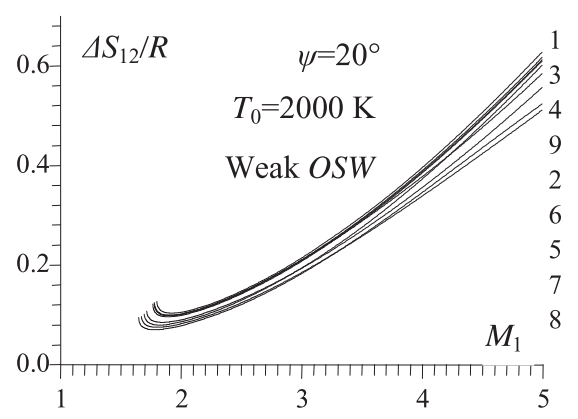

(g) : Variation of $\Delta S_{12} / R$ versus $M_{1}$.

FiguRE 10. Gas effect on the variation of $O S W$ parameters versus $M_{1}$. 


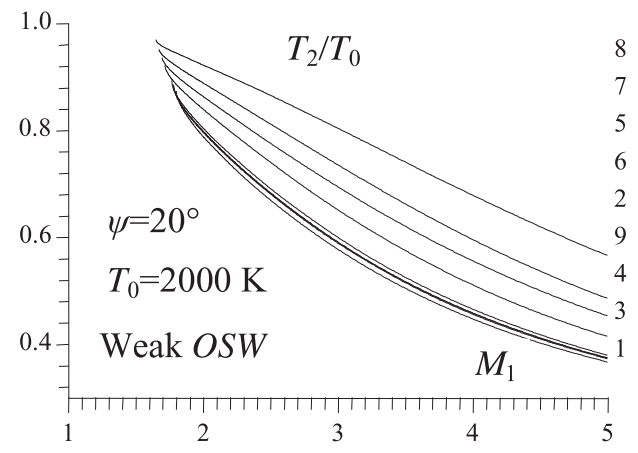

(a) : Variation of $T_{2} / T_{0}$ versus $M_{1}$.

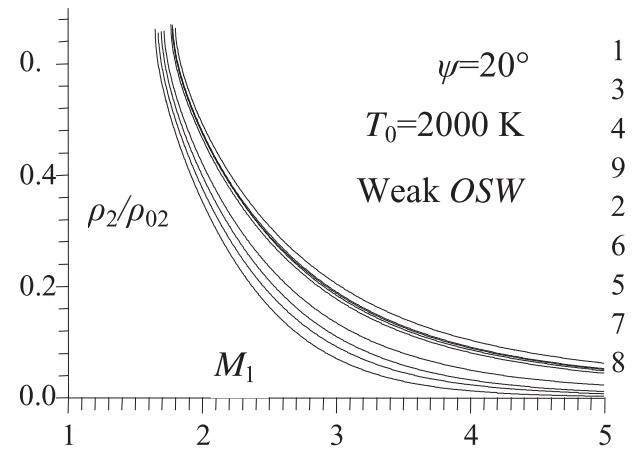

(b) : Variation of $\rho_{2} / \rho_{02}$ versus $M_{1}$.

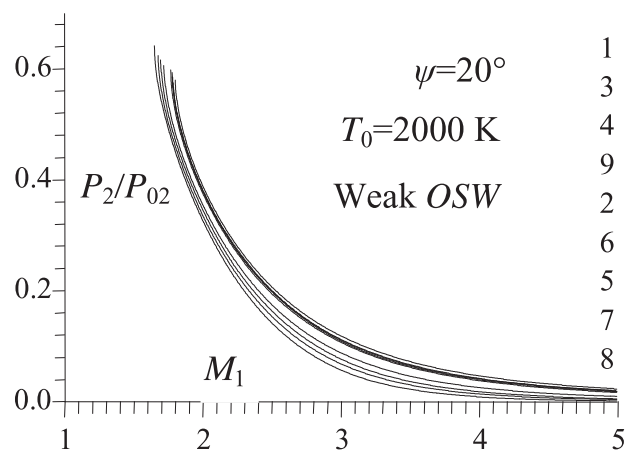

(c) : Variation of $P_{2} / P_{02}$ versus $M_{1}$.

Figure 11. Gas effect on the variation of the isentropic parameters behind $O S W$ versus $M_{1}$.

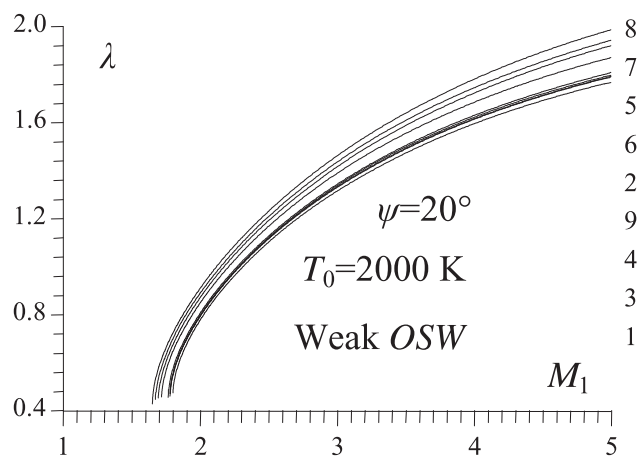

FiguRE 12. Gas effect on the variation of $\lambda$ versus $M_{1}$.

Figure 17 shows the gas effect on the variation of the adaptation distance $\lambda$ of an axially symmetrical air intake as a function of $\theta C$ for weak $C S W$ when $M_{1}=3.00$ and $T_{0}=2000 \mathrm{~K}$.

Figure 18 shows the gas effect on the variation of the wave drag $D$ on the cone as a function of $\theta C$ for weak $C S W$ when $M_{1}=3.00$ and $T_{0}=2000 \mathrm{~K}$. 


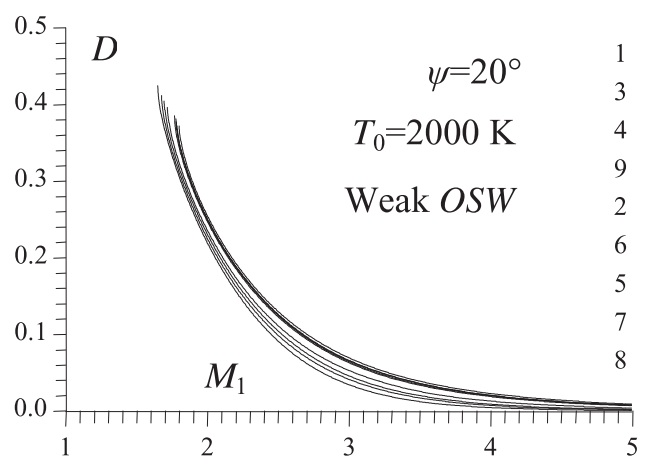

Figure 13. Gas effect on the variation of $D$ versus $M_{1}$.

TABLE 8. Gas effect on $\psi_{\max }$ for $C S W$ at $T_{0}=2000 \mathrm{~K}$.

\begin{tabular}{llllll}
\hline $\mathrm{N}$ & Gas & $M_{1}=2.00$ & $M_{1}=3.00$ & $M_{1}=4.00$ & $M_{1}=5.00$ \\
\hline 1 & $\mathrm{H}_{2}$ & 42.1447 & 51.2780 & 54.8117 & 56.4785 \\
2 & $\mathrm{O}_{2}$ & 42.5895 & 51.8591 & 55.4671 & 57.1827 \\
3 & $\mathrm{~N}_{2}$ & 42.1870 & 51.2433 & 54.7503 & 56.4203 \\
4 & $\mathrm{CO}$ & 42.2762 & 51.3571 & 54.8651 & 56.5309 \\
5 & $\mathrm{CO}_{2}$ & 45.0537 & 55.8338 & 60.2896 & 62.4749 \\
6 & $\mathrm{H}_{2} \mathrm{O}$ & 44.1917 & 54.2511 & 58.1804 & 60.0199 \\
7 & $\mathrm{NH}_{3}$ & 45.9271 & 57.1472 & 61.7199 & 63.8596 \\
8 & $\mathrm{CH}_{4}$ & 46.9346 & 58.9735 & 64.1480 & 66.6659 \\
9 & Air & 42.2863 & 51.5031 & 55.0319 & 56.6616 \\
\hline
\end{tabular}

TABle 9. Gas effect on $\psi_{\max }$ for $C S W$ when $M_{1}=3.00$.

\begin{tabular}{llllll}
\hline $\mathrm{N}$ & Gas & $T_{0}=298$ & $T_{0}=1000 \mathrm{~K}$ & $T_{0}=2000 \mathrm{~K}$ & $T_{0}=3000 \mathrm{~K}$ \\
\hline 1 & $\mathrm{H}_{2}$ & 48.9344 & 49.6048 & 51.2880 & 52.2598 \\
2 & $\mathrm{O}_{2}$ & 50.2401 & 50.7721 & 51.8591 & 52.7508 \\
3 & $\mathrm{~N}_{2}$ & 49.0980 & 49.4853 & 51.2433 & 52.1278 \\
4 & $\mathrm{CO}$ & 49.0769 & 50.2192 & 51.3571 & 52.3015 \\
5 & $\mathrm{CO}_{2}$ & 53.1021 & 54.6856 & 55.8338 & 56.6229 \\
6 & $\mathrm{H}_{2} \mathrm{O}$ & 50.8301 & 52.4581 & 54.2511 & 55.5577 \\
7 & $\mathrm{NH}_{3}$ & 53.3412 & 54.7138 & 57.1472 & 58.4789 \\
8 & $\mathrm{CH}_{4}$ & 54.7541 & 56.3990 & 58.9735 & 60.0808 \\
9 & Air & 49.2644 & 50.2367 & 51.5031 & 52.6540 \\
\hline
\end{tabular}

Figure 19 shows the gas effect on the variation of $\beta, \psi, M_{2}, T_{2} / T_{1}, \rho_{2} / \rho_{1}, P_{2} / P_{1}, P_{02} / P_{01}$ and $\Delta S 12 / R$ through a weak $C S W$ versus of $M_{1}$ for $\psi=20^{\circ}$ and $T_{0}=2000 \mathrm{~K}$. Only for $\beta, \psi$ and $M_{2}$ was added the variation for the strong shock.

Figure 20 shows the effect of gas on the variation of the isentropic parameters $T_{2} / T_{0}, \rho_{2} / \rho_{02}$ and $P_{2} / P_{02}$ after weak $C S W$ versus $M_{1}$ when $\psi=20^{\circ}$ and $T_{0}=2000 \mathrm{~K}$.

Figure 21 shows the effect of gas on the variation of the isentropic parameters $M_{C}, T_{C} / T_{0}, \rho_{C} / \rho_{02}$ and $P_{C} / P_{02}$ on the cone surface for weak $C S W$ versus $M_{1}$ when $\psi=20^{\circ}$ and $T_{0}=2000 \mathrm{~K}$. Only for $M_{C}$ was added the variation for strong shock.

Figure 22 shows the gas effect on the variation of the adaptation distance $\lambda$ of an axially symmetrical air intake as a function of $M_{1}$ for weak $C S W$ when $\psi=20^{\circ}$ and $T_{0}=2000 \mathrm{~K}$. 


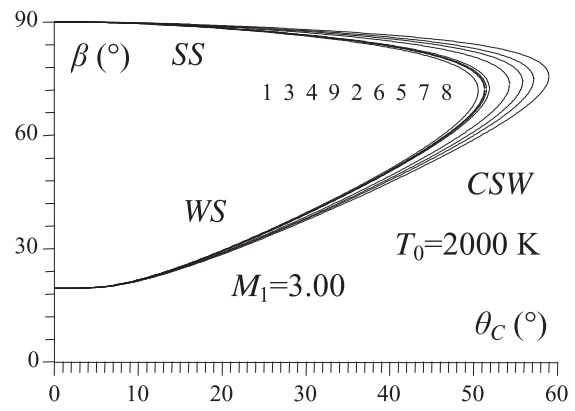

(a) : Variation of $\beta$ versus $\theta_{C}$.

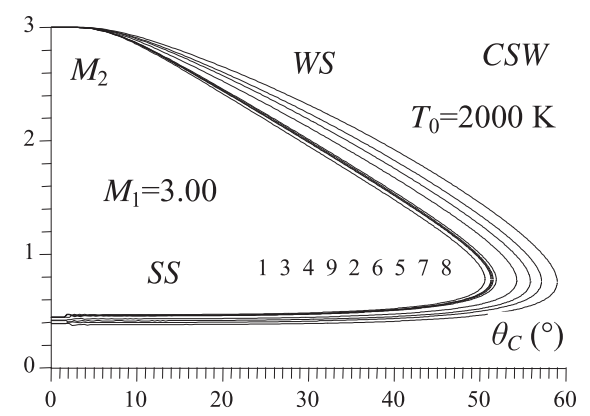

(c) : Variation of $M_{2}$ versus $\theta_{C}$.

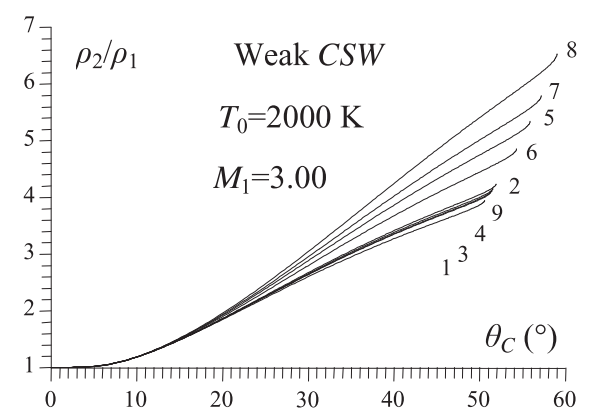

(e) : Variation of $\rho_{2} / \rho_{1}$ versus $\theta_{C}$.

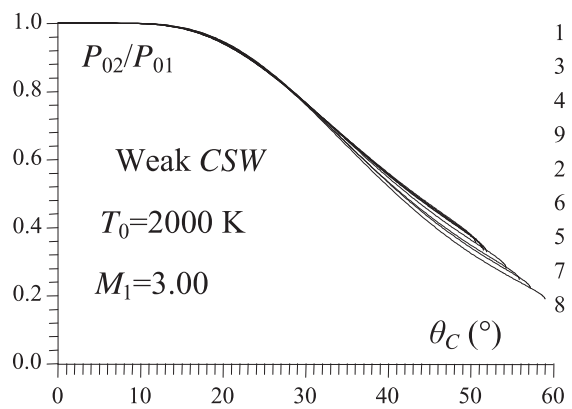

(g) : Variation of $P_{02} / P_{01}$ versus $\theta_{C}$.

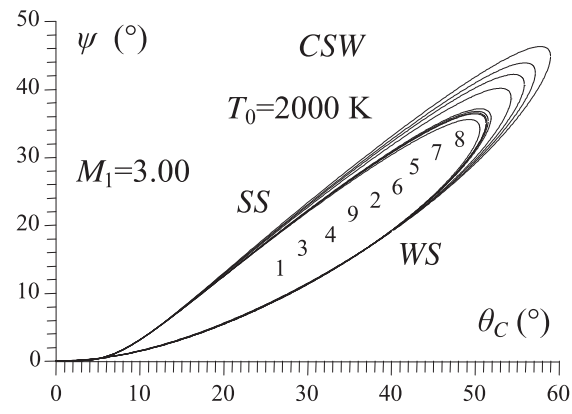

(b) : Variation of $\psi$ versus $\theta_{C}$.

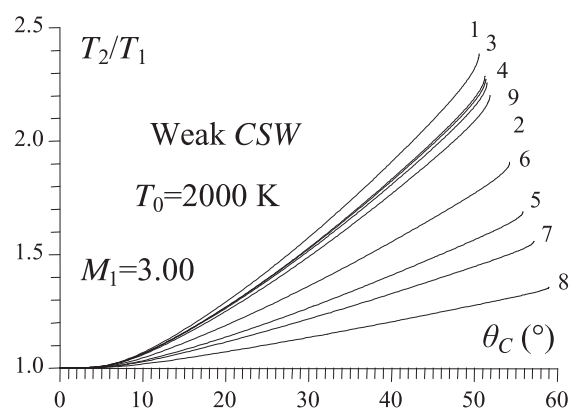

(d) : Variation of $T_{2} / T_{1}$ versus $\theta_{C}$.

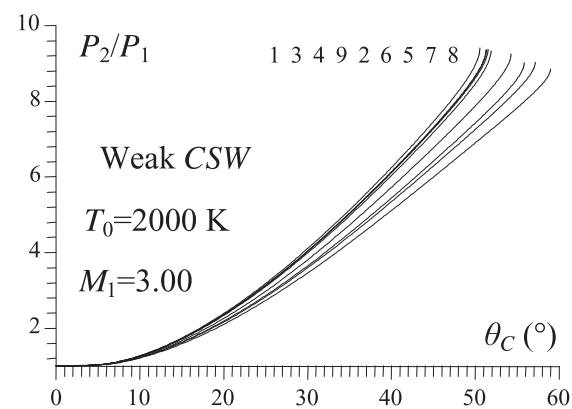

(f) : Variation of $P_{2} / P_{1}$ versus $\theta_{C}$.

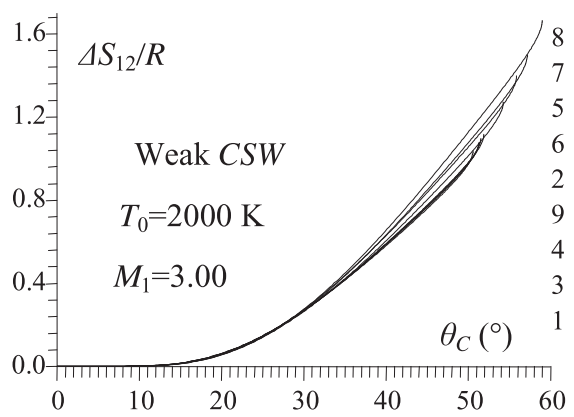

(h) : Variation of $\Delta S_{12} / R$ versus $\theta_{C}$.

Figure 14. Gas effect on the variation of $C S W$ parameters versus $\theta C$. 


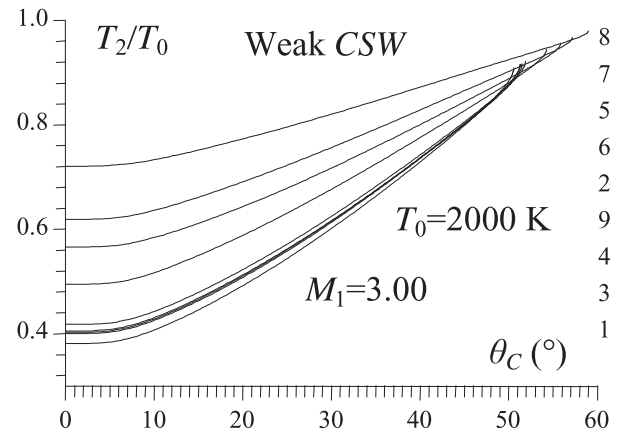

(a) : Variation of $T_{2} / T_{0}$ versus $\theta_{C}$.

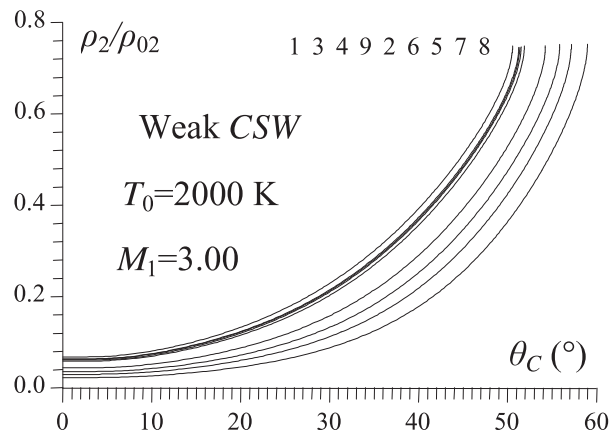

(b) : Variation of $\rho_{2} / \rho_{02}$ versus $\theta_{C}$.

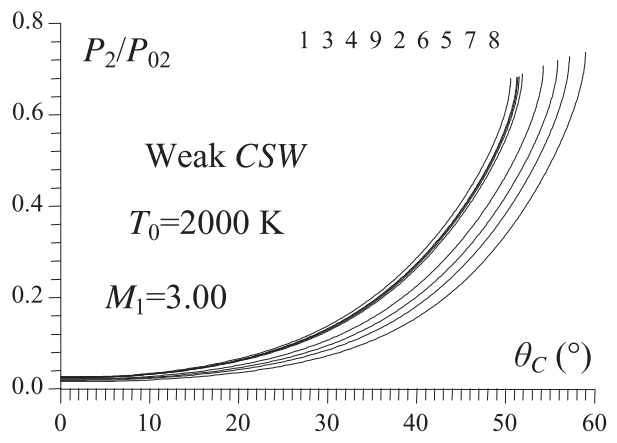

(c) : Variation of $P_{2} / P_{02}$ versus $\theta_{C}$.

FiguRE 15. Gas effect on the variation of isentropic parameters behind $C S W$ versus $\theta C$.

TABLE 10. Gas effect on $M_{\text {Lim }}$ for $C S W$ at $T_{0}=2000 \mathrm{~K}$.

\begin{tabular}{llllll}
\hline $\mathrm{N}$ & Gas & $\psi=10^{\circ}$ & $\psi=20^{\circ}$ & $\psi=30^{\circ}$ & $\psi=40^{\circ}$ \\
\hline 1 & $\mathrm{H}_{2}$ & 1.0508 & 1.2102 & 1.4503 & 1.8678 \\
2 & $\mathrm{O}_{2}$ & 1.0498 & 1.1982 & 1.4438 & 1.8470 \\
3 & $\mathrm{~N}_{2}$ & 1.0512 & 1.2117 & 1.4512 & 1.8696 \\
4 & $\mathrm{CO}$ & 1.0506 & 1.2078 & 1.4486 & 1.8629 \\
5 & $\mathrm{CO}_{2}$ & 1.0471 & 1.1865 & 1.4095 & 1.7491 \\
6 & $\mathrm{H}_{2} \mathrm{O}$ & 1.0479 & 1.1899 & 1.4198 & 1.7788 \\
7 & $\mathrm{NH}_{3}$ & 1.0462 & 1.1825 & 1.3985 & 1.7207 \\
8 & $\mathrm{CH}_{4}$ & 1.0453 & 1.1787 & 1.3878 & 1.6925 \\
9 & Air & 1.0503 & 1.2004 & 1.4477 & 1.8618 \\
\hline
\end{tabular}

Figure 23 shows the gas effect on the variation of the wave drag $D$ on the cone as a function of $M_{1}$ for weak $C S W$ when $\psi=20^{\circ}$ and $T_{0}=2000 \mathrm{~K}$.

\subsection{Comments}

It should first be noted that whatever the used gas, there is its influence on all the physical parameters for the $O S W$ and $C S W$ according to all the presented figures in this work. Generally we are interested in $W S$ for $O S W$ and $C S W$. The $N S W$ is of $S S$ type. 


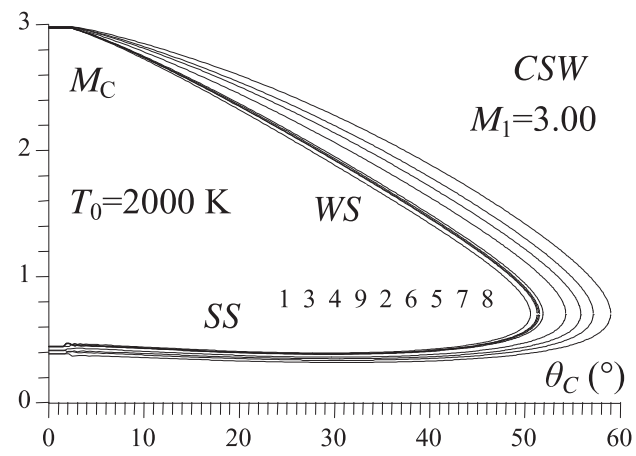

(a) : Variation of $M_{\mathrm{C}}$ versus $\theta_{C}$.

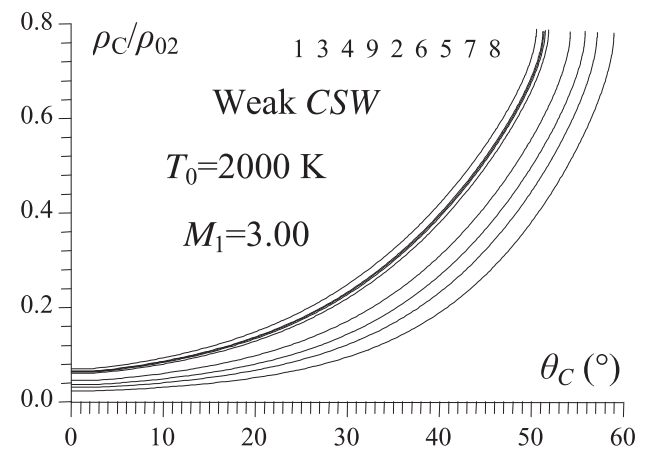

(c) : Variation of $\rho_{\mathrm{C}} / \rho_{02}$ versus $\theta_{C}$.

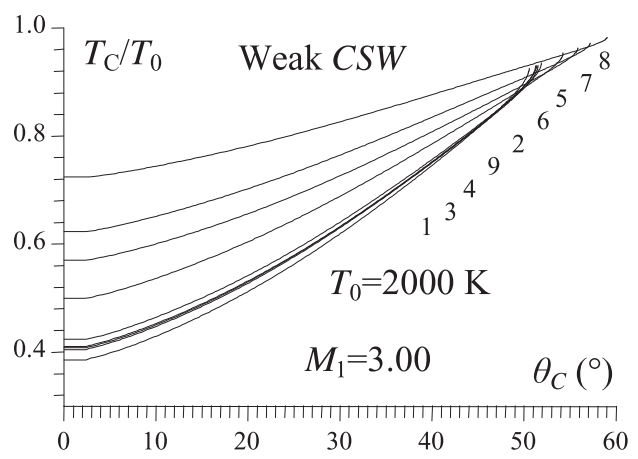

(b) : Variation of $T_{\mathrm{C}} / T_{0}$ versus $\theta_{C}$.

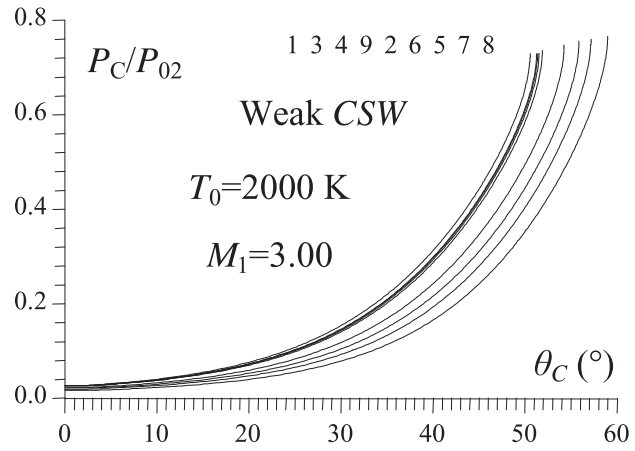

(d) : Variation of $P_{\mathrm{C}} / P_{02}$ versus $\theta_{C}$.

Figure 16. Gas effect on the variation of isentropic parameters on the cône surface versus $\theta C$.

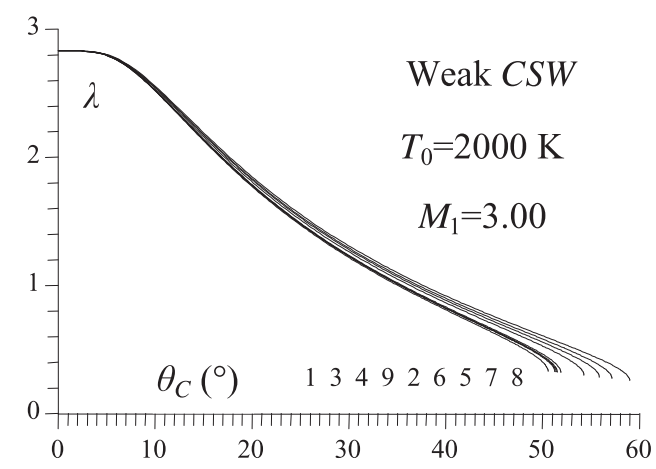

Figure 17. Gas effect on the variation of $\lambda$ versus $\theta C$.

Between $W S$ and $S S$, the inverse case is deduced on the influence rate of the gas on the variation of the parameters $M_{2}, P_{02} / P_{01}, \Delta S 12 / R, \rho_{2} / \rho_{02}$ and $P_{2} / P_{02}$ of $O S W$ and $C S W$, and for $\beta$ and $\lambda$ of $O S W$ and $C S W$, and for $\psi, \rho_{C} / \rho_{02}, P_{C} / P_{02}$ of $C S W$. While the same influence rate is observed for the parameters $T_{2} / T_{1}, \rho_{2} / \rho_{1}$, $P_{2} / P_{1}, T_{2} / T_{0}$ of $O S W$ and $C S W$, and for $D$ of $O S W$ and $C S W$, and for $T_{C} / T_{0}$ of $C S W$.

According to the gas dynamic, aeronautical and aerospace applications discussed in this paper, it can be said to choose the interested parameters for each application. 


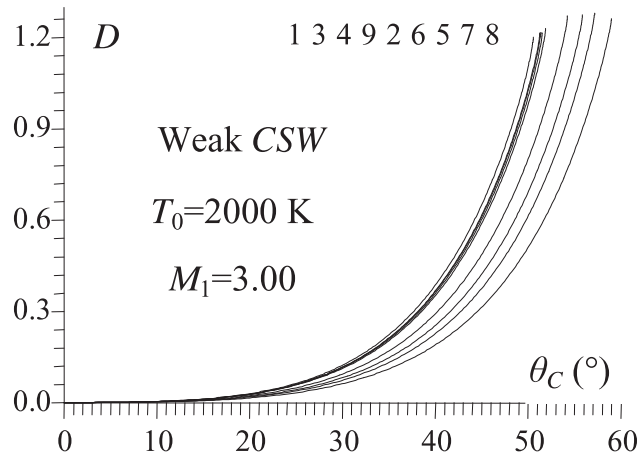

Figure 18. Gas effect on the variation of $D$ versus $\theta C$.

According to the 9 chosen gases presented in the Figures 6-22 and 23, it is noted that the influence of the gases $\mathrm{CH}_{4}, \mathrm{NH}_{3}, \mathrm{CO}_{2}, \mathrm{H}_{2} \mathrm{O}$ and $\mathrm{O}_{2}$ is of opposite direction to the behavior and the influence of the gases $\mathrm{H}_{2}$, $\mathrm{N}_{2}$, CO with respect to air. We also notice that the $\mathrm{O}_{2}$, despite being different in air, its behavior is very close compared to air with an error which does not exceed $1 \%$ at the maximum. We also note that the maximum deviation from air is found for the gas $\mathrm{CH}_{4}$, then $\mathrm{NH}_{3}$, then $\mathrm{CO}_{2}$ and $\mathrm{H}_{2} \mathrm{O}$. The smallest (closest) error is found for $\mathrm{O}_{2}$, although the 4 gases $\mathrm{H}_{2}, \mathrm{O}_{2}, \mathrm{~N}_{2}$ and $\mathrm{CO}$ give a very small deviation from air, which gives the possibility of replacing air with one of these 4 gases, giving a very small change in the state of the flow.

For drag force (Figs. 9, 13, 18 and 23), the $\mathrm{CH}_{4}$ becomes the right choice since there is a decrease in $D$ of the engine. The bad is $\mathrm{H}_{2}$. For a cruise flight, it is best to have the smallest possible $D$. While landing or taking off, it's best to have as much drag as possible. We know that there are other maneuvers to increase or decrease this drag. But gas also has its own influence.

The flow around a pointed airfoil is characterized by the birth of an $O S W$ at the leading edge then a series of progressive compression or expansion through the upper and lower surfaces. If there is an increase in the inclination of the wall, then we will have a compression of the flow and this study will be used to determine the local parameters of the flow. If there is a decrease in the inclination of the wall compared to the upstream flow, we will have in this case an expansion and the parameters of the flow will be determined by the use of the Prandtl Meyer function at $H T[6,26]$. To see the interest of the gas, we added the aerodynamic fineness $\left(\chi=C_{y} / C_{x}\right)$ for each gas. For $C_{x}, C_{y}$ and $C_{m}$ presented in the Table 6 for the airfoil of Figure 5 , we must see the aerodynamic finesse which is observed maximum for $\mathrm{H}_{2}$ and minimum for $\mathrm{CH}_{4}$. In this case the right choice is $\mathrm{H}_{2} . \mathrm{O}_{2}, \mathrm{~N}_{2}, \mathrm{CO}$ gases are also good choices. $\mathrm{CO}_{2}, \mathrm{H}_{2} \mathrm{O}, \mathrm{NH}_{3}$ and $\mathrm{CH}_{4}$ are bad choices. To make a comparison, we added the results for air which are presented in [26].

The values presented in Table 6 vary with $M_{1}, T_{0}$ and $\alpha$ as well as the used gas. All of these and other parameters will be the goal of future work.

In all $O S W$ and $C S W$ figures results (figures and tables), and for $M_{1}<1.50$ approximately, the choice of such a gas propulsion poses no importance, since the setting through the shock are almost the same.

It should be noted that for $O S W$ and $C S W$, we have the possibility of having a detached shock wave according to the Tables 2 and 3 for $O S W$ and the Tables 8 and 9 for $C S W$. According to Figures 6, 7, 8 and 9 for $O S W$ and Figures 15, 16, 17 and 18 for $C S W$, we have no solution depending on the model of the attached shock wave. We must to solve the Euler equation to detect the detached shock wave. The precise value of $M_{1}$ between attached and detached shock is given in Tables 4 and 5 for the $O S W$ and Tables 10 and 11 for the $C S W$.

In all $O S W$ and $C S W$ figures results (figures and tables), if $\varphi<10^{\circ}$ approximately, the choice of gas also has none, since the curves are almost confused. We can calculate the errors between each curves and curve 9 for air.

On Table 7 and according to the Mach number obtained in the three zones 2, 3 and 4, we notice a remarkable degradation of the Mach number after each reflection. Consequently there is also disadvantaged degradation of the other physical parameters which make the engine unreliable for aerodynamic flight characteristics. So to 


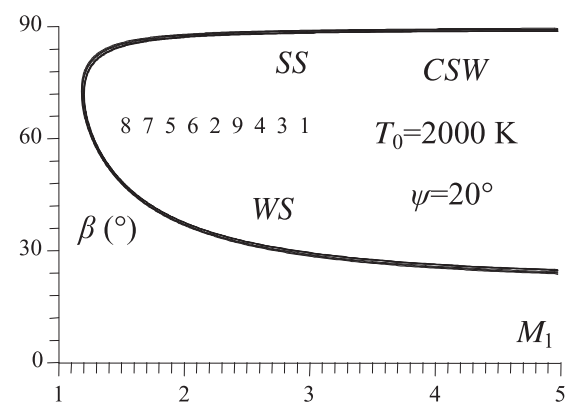

(a) : Variation of $\beta$ versus $M_{1}$.

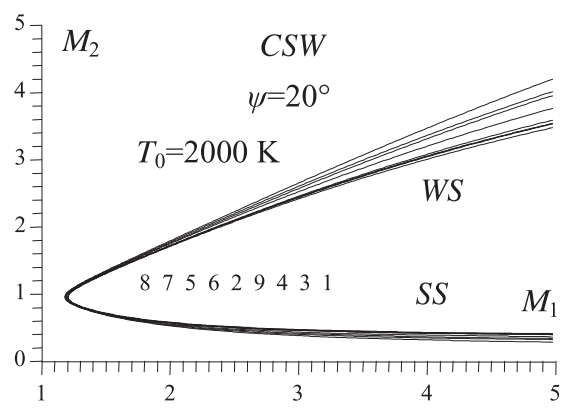

(c) : Variation of $M_{2}$ versus $M_{1}$.

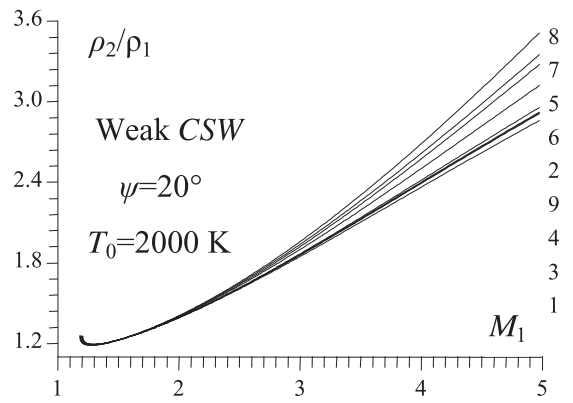

(e) : Variation of $\rho_{2} / \rho_{1}$ versus $M_{1}$.

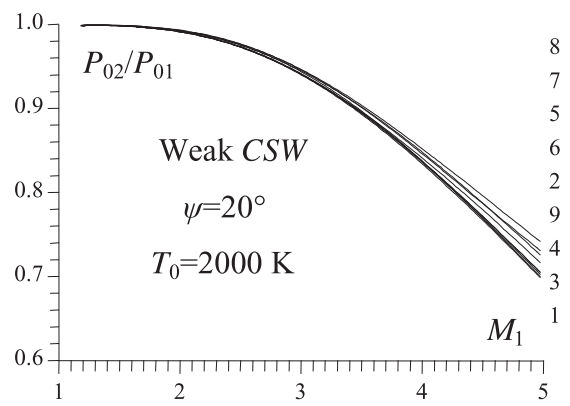

(g) : Variation of $P_{02} / P_{01}$ versus $M_{1}$.

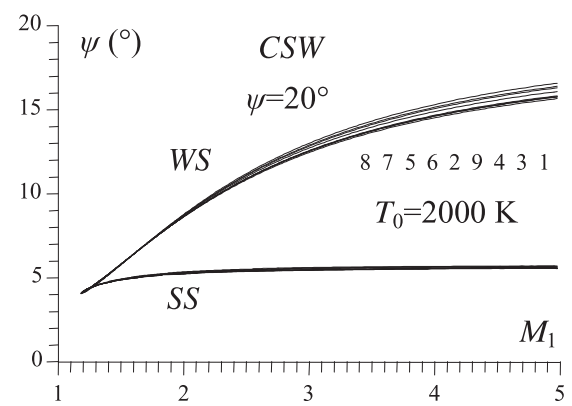

(b) : Variation of $\psi$ versus $M_{1}$.

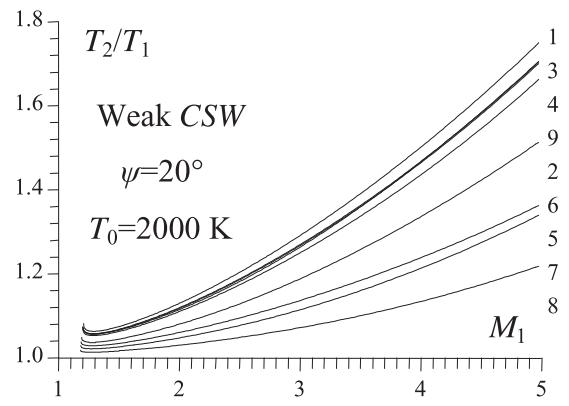

(d) : Variation of $T_{2} / T_{1}$ versus $M_{1}$.

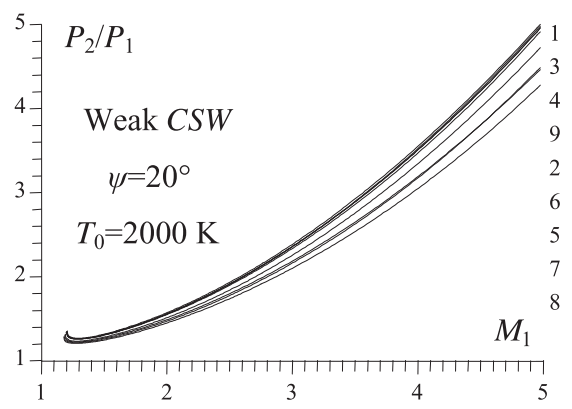

(f) : Variation of $P_{2} / P_{1}$ versus $M_{1}$.

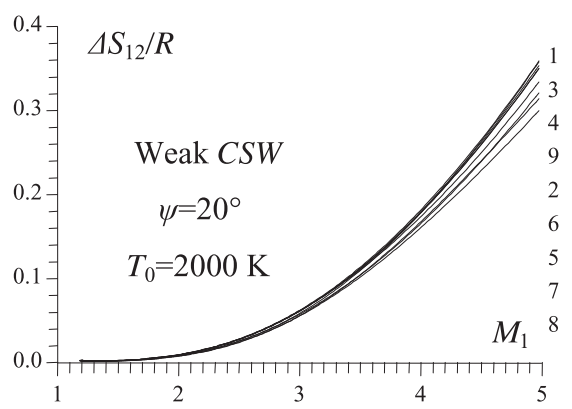

(h) : Variation of $\Delta S_{12} / R$ versus $M_{1}$.

Figure 19. Gas effect on the variation of $C S W$ parameters versus $M_{1}$. 


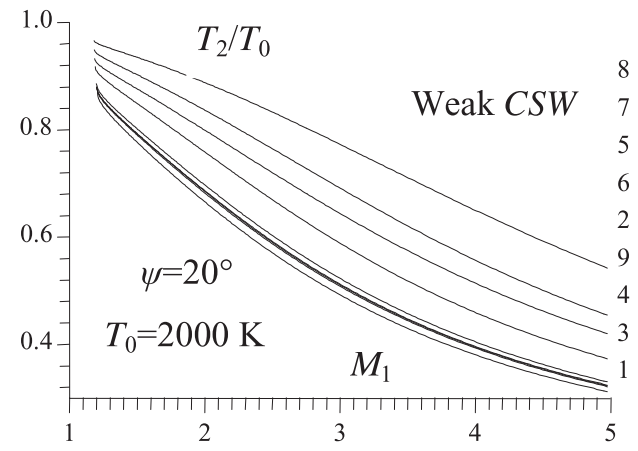

(a) : Variation of $T_{2} / T_{0}$ versus $M_{1}$.

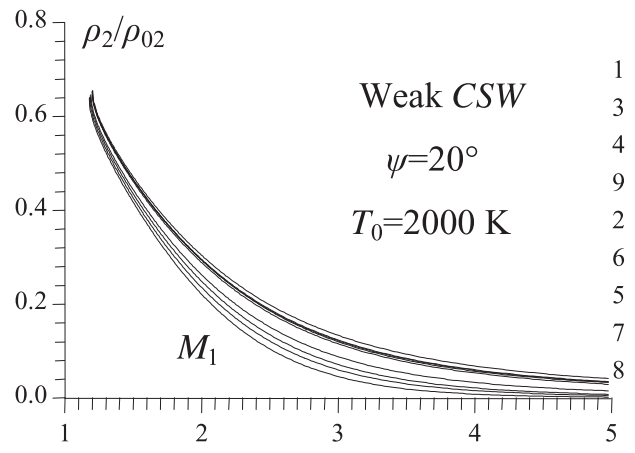

(b) : Variation of $\rho_{2} / \rho_{02}$ versus $M_{1}$.

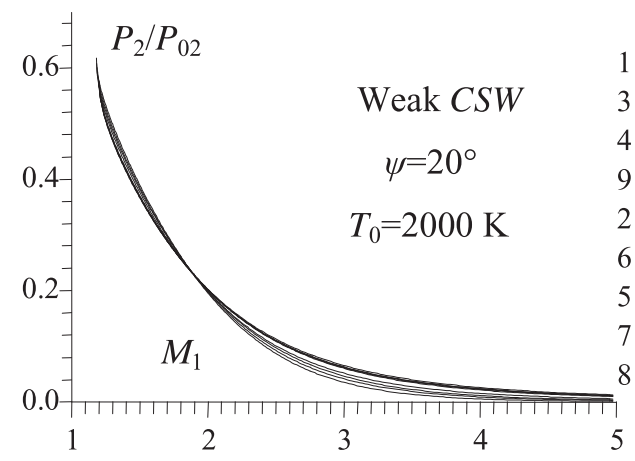

(c) : Variation of $P_{2} / P_{02}$ versus $M_{1}$.

FiguRE 20. Gas effect on the variation of the isentropic parameters behind CSW versus $M_{1}$.

TABle 11. Gas effect on $M_{l i m}$ for $C S W$ when $\psi=20^{\circ}$.

\begin{tabular}{llllll}
\hline $\mathrm{N}$ & Gas & $T_{0}=298$ & $T_{0}=1000 \mathrm{~K}$ & $T_{0}=2000 \mathrm{~K}$ & $T_{0}=3000 \mathrm{~K}$ \\
\hline 1 & $\mathrm{H}_{2}$ & 1.2124 & 1.2080 & 1.2089 & 1.1993 \\
2 & $\mathrm{O}_{2}$ & 1.2107 & 1.2030 & 1.1982 & 1.1948 \\
3 & $\mathrm{~N}_{2}$ & 1.2129 & 1.2093 & 1.2099 & 1.1998 \\
4 & $\mathrm{CO}$ & 1.2119 & 1.2050 & 1.2079 & 1.1984 \\
5 & $\mathrm{CO}_{2}$ & 1.1943 & 1.1897 & 1.1865 & 1.1843 \\
6 & $\mathrm{H}_{2} \mathrm{O}$ & 1.2035 & 1.1960 & 1.1899 & 1.1863 \\
7 & $\mathrm{NH}_{3}$ & 1.1927 & 1.1885 & 1.1825 & 1.1797 \\
8 & $\mathrm{CH}_{4}$ & 1.1904 & 1.1839 & 1.1787 & 1.1766 \\
9 & Air & 1.2111 & 1.2040 & 1.2004 & 1.1973 \\
\hline
\end{tabular}

avoid this situation, it is necessary to adapt the intake according to Figure 3 and to move the central body by a distance $\lambda$ according to the relation (6.1) for each gas, giving the result presented on Figures 8 and 12. The effect of gas remains the same for an $O S W$ configuration discussed previously.

The temperature $T_{0}$ also influences the parameters of $O S W$ and $C S W$. In this study, we took the presentation of the results for $T_{0}=2000 \mathrm{~K}$. We did not prefer to vary the value of $T_{0}$ in the form of a graph, except in Tables 3 and 5 for $O S W$, Tables 9 and 11 for $C S W$ for aim to calculate $\psi M a x$ and MLim respectively. It should be noted that our application is designed at HT. The $P G$ model does not depend on $T_{0}$, and our $H T$ model becomes a generalization of the $P G$ model presented in $[2,3,13,16,17,22,25-27]$. 


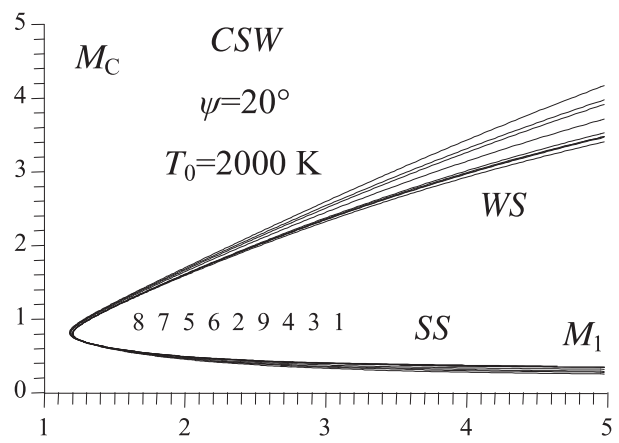

(a) : Variation of $M_{\mathrm{C}}$ versus $M_{1}$.

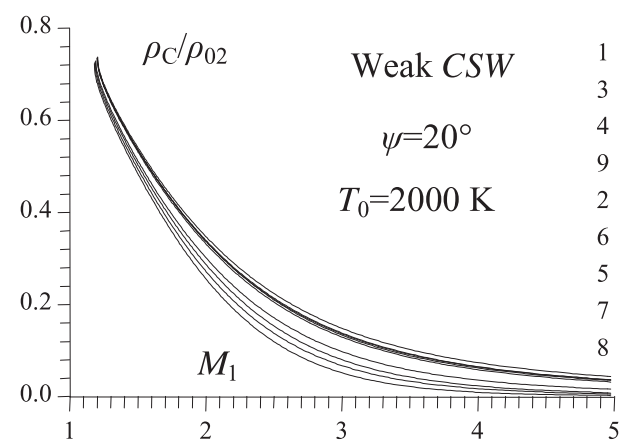

(c) : Variation of $\rho_{\mathrm{C}} / \rho_{02}$ versus $M_{1}$.

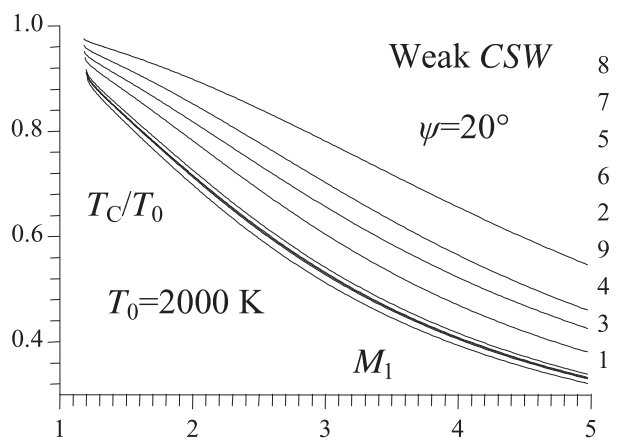

(b) : Variation of $T_{\mathrm{C}} / T_{0}$ versus $M_{1}$.

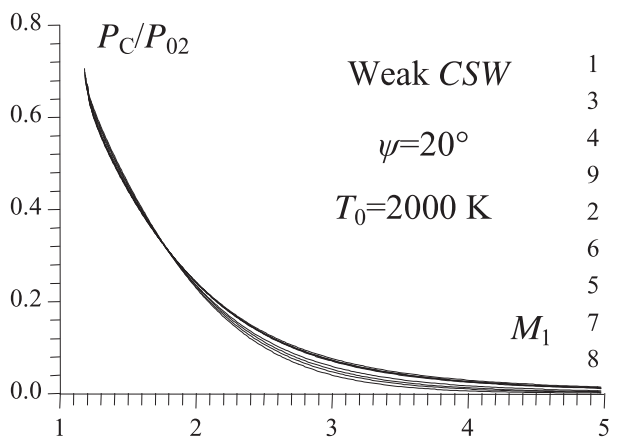

(d) : Variation of $P_{\mathrm{C}} / P_{02}$ versus $M_{1}$.

FiguRE 21. Gas effect on the variation of isentropic parameters on the cône surface versus $M_{1}$.

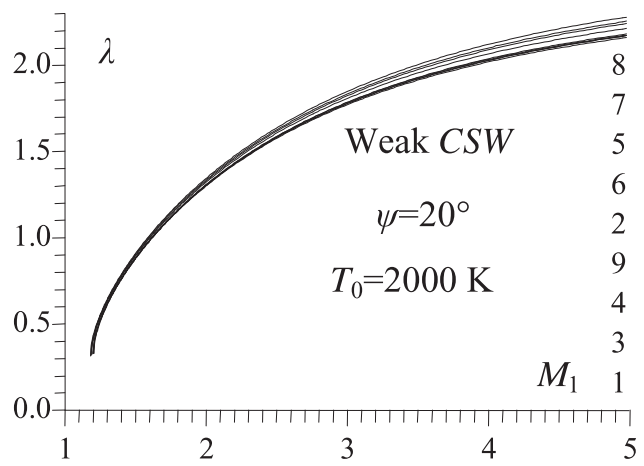

Figure 22. Gas effect on the variation of $\lambda$ versus $M_{1}$.

Due to the spread of waste and its combustion in the world with the increasing density for several years, the random cutting of trees that is harmful to the environment and the expectations that will occur in the future, we expect that the basic composition of the air will not return like that composed according to the Table $1[4,10,14,20,21,32]$. For this reason it is necessary to take samples of the air in order to correct the thermodynamics of air in ambient medium in order to determine a correction at $C_{P}(T)$ and $R$.

We can use a gas instead of air having better performance compared to air after studying this gas according to our work for applications of aerodynamics (gas dynamic) external by blowing gas at the nose of the obstacle 


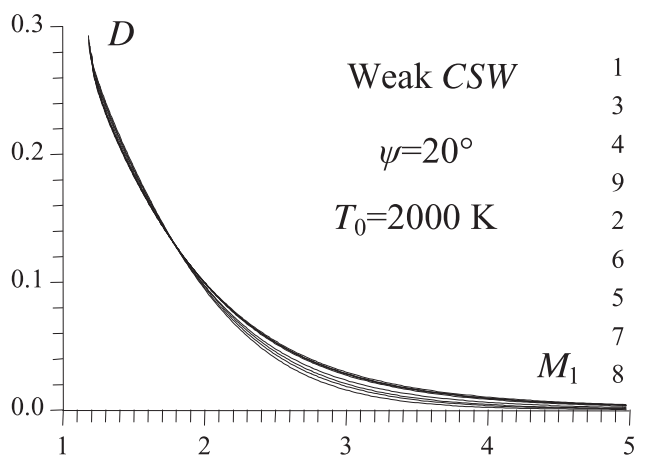

Figure 23. Gas effect on the variation of $D$ versus $M_{1}$.

with a suitable flow rate making it possible to influence the inclination of the shock and consequently on all the parameters of shock, which gives us an gas dynamic acceleration or deceleration without mechanical activation.

The case of dissociation can be studied in order to see the possibility of finding results in hypersonic regime. In this case it is necessary to calculate the specific heat at constant pressure of the atoms constituting the dissociated gas according to the relation of the dissociation and to inject the resulting formula in the numerical program.

\section{Conclusion}

This work allows us to study the effect of use of the propellant gases on the thermodynamic parameters through an $O S W$ and $C S W$. Can be drawn from the following conclusions:

- Numerical computation program can do any gas found in nature. It should be added in this case the variation of the specific heat $C_{P}(T)$ and the gas constant $R$ even calculation of the function $H(T)$.

- The $C_{P}(T)$ function, $R$ and $\gamma(T)$ ratio characterize the calorific value of the gas and mainly affects all parameters across the shock.

- The famous relation between $\left(\beta, \varphi, M_{1}\right)$ at $H T$ for $O S W$ and $C S W$ has been developed implicitly and numerically with great precision.

- The stagnation temperature and the Mach number also affect all parameters through the shock.

- The higher the ratio $\gamma$ of the gas is high, the higher $M_{2}$ is very small also affects all other parameters. The jump of entropy in this case is big enough yet interpreted by the high load losses affecting for example the sound of the shock wave.

- The higher the ratio $\gamma(T)$ of the gases, the smaller the shock parameters. This type of gas is called by cold gas, this is the case for $\mathrm{H}_{2}, \mathrm{O}_{2}, \mathrm{~N}_{2}, \mathrm{CO}$ and air.

- The $P G$ model at low temperature considered for constant $C_{P}$ becomes a particular case of our study at $H T$.

- It is recommended to take air samples every some years to correct the thermodynamics of the air in terms of $C_{P}(T)$ and $R$.

- We can use a gas instead of air having better performance compared to air after studying this gas according to our work for applications of aerodynamics (gas dynamic) external by blowing gas at the nose of the obstacle with a suitable flow rate making it possible to influence the inclination of the shock and consequently on all the parameters of shock.

- If we keep the same common parameter between gas and air and especially drag force, the use of another gas instead of air will improve or de-improve the use of this gas. Among the gases chosen, the use of $\mathrm{H}_{2}$, $\mathrm{N}_{2}$, CO by increasing $M_{2}, D$ and the use of gases $\mathrm{CH}_{4}, \mathrm{NH}_{3}, \mathrm{CO}_{2}$ and $\mathrm{H}_{2} \mathrm{O}$ will degrade $M_{2}$ and $D$. 
- At low $T$ and $M$, the difference in results between the different gases can be neglected, which shows the possibility of using any gas instead of air. We can go up to $M_{1}=1.50$ regardless of $T_{0}$ and $\varphi$, or $T_{0}<240$ $\mathrm{K}$ regardless of $M_{1}$ and $\varphi$, or $\varphi<10^{\circ}$ regardless of $T_{0}$ and $M_{1}$.

- The use of gases such as $\mathrm{CH}_{4}, \mathrm{NH}_{3}, \mathrm{H}_{2} \mathrm{O}$ and $\mathrm{CO}_{2}$ degrades $\mathrm{M}_{2}$ compared to that of air. While $\mathrm{H}_{2}, \mathrm{~N}_{2}$ and $\mathrm{CO}$ increases M2 compared to air, especially when $T_{0}$ is high.

- It is recommended to take samples of air from the friendly medium every certain year in order to correct the thermodynamics of the air in terms of $C_{P}(T)$ and $R$ and chemical composition.

- The case of dissociation can be studied in order to see the possibility of finding results in hypersonic regime. In this case it is necessary to calculate the specific heat at constant pressure of the atoms constituting the dissociated gas according to the relation of the dissociation and to inject the resulting formula in the numerical program.

As perspectives, we can study our problem by the use of CFD or even make experimental tests by supersonic wind tunnel.

We can use the present work to study the effect of gas propulsion at $H T$ to calculating the detached shock around a blunt body or pointed bodies with large wall inclination, or low flow Mach number, and to find the aerodynamic and gas dynamics coefficients and shock parameters.

\section{NomenClature}

$\begin{array}{ll}C_{P} & \text { Specific heat at constant pressure } \\ a & \text { Sound velocity. } \\ H & \text { Enthalpy. } \\ M & \text { Mach number. } \\ \theta & \text { Polar radius deviation for } C S W \\ \theta C & \text { Cone deviation } \\ P & \text { Static pressure } \\ R & \text { Thermodynamic constant of gas } \\ T & \text { Temperature } \\ V & \text { Gas velocity } \\ r & \text { Polar ray } \\ \gamma & \text { Specific heats ratio } \\ \rho & \text { Density } \\ a^{\prime}, b^{\prime}, c^{\prime} & \text { Coefficients of } C_{P}(T) \text {. See equation (1.1) } \\ \psi & \text { Dihedron deviation } \\ \beta & \text { Shock wave deviation } \\ L & \text { Longitudinal length of the dihedral } \\ l & \text { Unit of dihedral depth } \\ \Delta S & \text { Entropy jump } \\ h & \text { Half height of the air intake nacelle } \\ C & \text { Airfoil chord } \\ d & \text { Adaptation distance of a supersonic air intake } \\ \lambda & \text { Standardized air intake adaptation distance } \\ \sigma & \text { Shock wave reflection with respect to the horizontal wall } \\ D & \text { Drag of the dihedron } \\ H T & \text { High Temperature } \\ P G & \text { Perfect Gas } \\ N S W & \text { Normal Shock Wave } \\ & \end{array}$


$O S W \quad$ Oblique Shock Wave

$C S W \quad$ Conical Shock wave

SS Strong Shock

WS Weak Shock

$f_{E}(x) \quad$ Equation of the Extrados airfoil

$f_{I}(x) \quad$ Equation of the Intrados airfoil

$\alpha \quad$ Incidence angle of an airfoil

$C_{x} \quad$ Drag coefficient of the airfoil

$C_{y} \quad$ Lift coefficient of the airfoil

$C_{m} \quad$ Pitching moment coefficient of the airfoil

$k \quad k=1$ for dihedron and $k=2$ for the cone

$\varphi \quad \varphi=\psi$ for dihedron and $\varphi=\theta C$ for the cone

$\omega \omega=2$ for dihedron and $\omega=\pi$ for cone

$\chi \quad$ Aerodynamic finesse of an airfoil

CFD Computational Fluid Dynamic

\section{Indices}

$0_{1}$

$0_{2}$

0

1

2

3

4

$S$

C

$r$

$\theta$

Max

Stagnation conditions before the shock

Stagnation conditions behind the shock

Stagnation condition

Parameter just before the shock wave

Parameter just after the shock wave

Parameter in the zone 3 of Figure 5

Parameter in the zone 4 of the Figure 5

Shock

Parameter on the cone surface

In radial direction

Lim

According to the transverse direction

Maximum

Lower limit

a

Ambient condition

\section{REFERENCES}

[1] A.M. Agnone, Approximations for weak and strong oblique shock wave angles. AIAA J. 32 (1994) 1543-1545.

[2] J.D. Anderson Jr., Hypersonic and High Temperature Gas Dynamics. McGraw-Hill Book Company, New York (1989).

[3] J. Anderson Jr., Modern Compressible Flow: With Historical Perspective. McGraw Hill Book Company, New York (1982).

[4] I. Barin, F. Sauer, R.E. Schultze and W.S. Sheng, Thermochemical Data on Pure Substances, Pt. 1, VCH Publishers, Wiesbaden, Germany (1989).

[5] M. Boun-jad, T. Zebbiche and A. Allali, Gas effect at high temperature on the supersonic nozzle conception. Int. J. Aeronaut. Space Sci. 18 (2017) 82-90.

[6] M. Boun-jad, T. Zebbiche and A. Allali, High temperature gas effect on the Prandtl-Meyer function with application for supersonic nozzle design. Mech. Ind. 18 (2017) 219.

[7] M. Boun-jad, T. Zebbiche and A. Allali, High temperature gas effect on the supersonic axisymetric minimum length Nozzle design. Int. J. Eng. Tech. Res. 07 (2017) 23-30.

[8] M. Boun-jad, T. Zebbiche and A. Allali, Numerical study of gas effect at high temperature on the supersonic plug and expansion deflexion nozzles design. Int. Res. J. Eng. Technol. 04 (2017) 1480-1488.

[9] M. Boun-jad and T. Zebbiche, High temperature gas effect on the normal shock wave parameters. Int. J. Mech. Prod. Eng. 5 (2017) $78-85$.

[10] A. Burcat and B. McBride, Ideal Gas Thermodynamic Data for Compounds Used in Combustion and Air-Pollution, TAE 675, Technion Israel Institute of Technology, Haifa, Israel (1992).

[11] A.R. Curtis, High-ordre Explicit Runge Kutta Formulae. Their uses, and limitations. J. Inst. Math. Appl. 16 (1975) 35-55.

[12] T. Elaichi and T. Zebbiche, Stagnation temperature effect on the conical shock with application for air. Chin. J. Aeronaut. 31 (2018) 672-697. 
[13] E.L. Goldsmith and J. Seddon, Intake Aerodynamics, 2nd edn. Blackwell Science (1999).

[14] W.M. Haynes, CRC Handbook of Chemistry and Physics, 93ème edn. CRC Press/Taylor and Francis, Boca Raton (2012).

[15] A. Iserles, A First Course in the Numerical Analysis of Differential Equations. Cambridge University Press (1996).

[16] E.T. Kenneth, Computation of Thermally Perfect Properties of Oblique Shock Waves, NASA CR-4749 (1996).

[17] E.T. Kenneth, Computation of thermally perfect oblique shcok waves properties, in AIAA-97-0868, 35th Aerospace Sciences Meeting and Exhibit, Aerospace Sciences Meetings, 06-09 January (1997).

[18] Z. Kopal, Tables of Supersonic Flow Around Cones. Massachusetts Institute of Technology, Dept. of Electrical Engineering Tech. Report No. 1, Cambridge, Mass. (1947).

[19] J.W. Maccoll, The conical shock wave formed by a cone moving at high speed, Proc. Roy. Soc. London A 159 (1937) 459-472.

[20] B.J. McBride, S. Gordon and M.A. Reno, Coefficients for Calculating Thermodynamic and Transport Properties of Individual Species, NASA TM 4513 (1993).

[21] B.J. McBride, S. Gordon and M.A. Reno, Thermodynamic data for fifty reference elements, NASA TP-3287 (1993).

[22] C.R. Peterson and P.G. Hill, Mechanics and Thermodynamics of Propulsion. Addition-Wesley Publishing Company Inc., New York, USA (1965).

[23] A. Ralston and P. Rabinowitz, A First Course in Numerical Analyses. McGraw-Hill Book Company (1985).

[24] J.L. Sims, Tables for Supersonic Flow Around Right Circular Cones at Zero Angle of Attack, NASA SP-3004 (1964).

[25] G.P. Sutton and O. Biblarz, Rocket Propulsion Elements, 8éme édition. John Wiley and Sons (2010).

[26] R. Takhnouni, T. Yahiaoui and A. Allali, Stagnation temperature effect on the supersonic flow around pointed airfoils with application for air. Arab. J. Sci. Eng. 44 (2019) 1185-1203.

[27] K.E. Tatum, Computation of thermally perfect oblique shock wave properties, in 35th Aerospace Sciences Meeting and Exhibit, Reno, AIAA-97-0868 (1997).

[28] G. Xiang et al., Cellular aluminum particle-air detonation based on realistic heat capacity model. Combust. Sci. Technol. 192 (2020) 1931-1945.

[29] G. Xiang et al., Numerical study on transition structures of oblique detonations with expansion wave from finite-length cowl. Phys. Fluids 32 (2020) 056108.

[30] G. Xiang et al., Study of the features of oblique detonation induced by a finite wedge in hydrogen-air mixtures with varying equivalence ratios. Fuel $\mathbf{2 6 4}$ (2020) 116854.

[31] T. Zebbiche, Effect of stagnation temperature on the normal shock wave. Int. J. Aeronaut. Space Sci. 10 (2009) 1-14.

[32] T. Zebbiche and Z. Youbi, Effect of stagnation temperature on supersonic flow parameters. Application for air in nozzles. Aeronaut. J. 111 (2007) 31-40. 